\title{
Flux pinning and boundary nucleation of vorticity in a mean field model of superconducting vortices
}

\author{
Charles M. ElliotT
}

Centre for Mathematical Analysis and its Applications, School of Mathematical Sciences, University of Sussex, Brighton BN1 9QH, UK

AND

VANESSA STYLES ${ }^{\dagger}$

School of Computing and Mathematical Sciences, Oxford Brookes University, Headington, Oxford OX 3 OBP, UK

[Received 17 December 1998 and in revised form 11 June 1999]

\begin{abstract}
We study a one-dimensional mean field model of superconducting vortices with a finite London penetration depth, flux pinning and nucleation of vorticity at inflow boundary sections. The existence of a unique weak solution is proved and the long time behaviour is studied. A numerical discretization of the model is derived and it is shown that as the time step and the mesh size tend to zero, the discrete solution converges to the unique weak solution of the continuous model. Some numerical computations are presented which illustrate the effects of flux pinning and the finite penetration depth.
\end{abstract}

\section{Introduction}

We begin with a brief introduction to the three space dimensional mean field model of superconducting vortices for a Type-II superconductor in its mixed state, derived in [4]. In the domain occupied by the superconductor we have

$$
\begin{gathered}
\mathbf{w}_{t}+\operatorname{curl}(\mathbf{w} \wedge \mathbf{v})=\mathbf{0}, \\
\lambda^{2}\left(\operatorname{curl}^{2} \mathbf{H}+\mathbf{H}=\mathbf{w},\right. \\
\nabla \cdot \mathbf{H}=0, \\
\operatorname{curl} \mathbf{H}=\mathbf{J}, \\
\mathbf{v}=(\mathbf{J} \times \hat{\mathbf{w}}) \frac{f(|\mathbf{J}|)}{|\mathbf{J}|},
\end{gathered}
$$

while in the region external to the superconductor we have

$$
\begin{array}{r}
\operatorname{curl} \mathbf{H}=\mathbf{J}, \\
\operatorname{curl} \mathbf{E}+\frac{\partial \mathbf{H}}{\partial t}=\mathbf{0},
\end{array}
$$

\footnotetext{
${ }^{\dagger}$ Email: vmstyles@brookes.ac.uk
} 


$$
\begin{aligned}
\nabla \cdot \mathbf{H} & =0, \\
\nabla \cdot \mathbf{E} & =\rho .
\end{aligned}
$$

Here $\mathbf{w}, \mathbf{v}, \mathbf{H}, \mathbf{J}, \mathbf{E}$ and $\rho$, respectively, denote vorticity, the velocity of the vorticity, average magnetic field, current, electric field and charge density. Furthermore, $\lambda$ is a length-scale relating to the penetration depth, $\hat{\mathbf{w}}$ denotes a unit vector in the direction of $\mathbf{w}$ and gives the local direction of the averaged flux lines. The magnitude $|\mathbf{w}|$ of $\mathbf{w}$ gives the density of flux lines. This is one model in a hierarchy of mathematical models of superconductors. Starting from the Ginzburg-Landau equations we can obtain in a high Ginzburg-Landau parameter limit, the London equation

$$
\lambda^{2} \operatorname{curl}^{2} \mathbf{H}_{i}+\mathbf{H}_{i}=\sum_{j \neq i} \vec{\delta}_{\Gamma_{j}},
$$

coupled with

$$
\mathbf{V}_{i}=\operatorname{curl} \mathbf{H}_{i} \times \vec{\tau}_{i},
$$

for the motion of line vortices [5]. Here $\Gamma_{i}$ is an evolving line vortex and $\vec{\delta}_{\Gamma_{i}}$ is the Dirac measure on $\Gamma_{i}$; $\mathbf{V}_{i}$ and $\tau_{i}$ denote the velocity and the unit tangent of $\Gamma_{i}$. The medium is in the normal state on the vortex lines and in the superconducting state elsewhere. Since there are many millions of vortices in a sample of appreciable size it is appropriate to carry out an averaging procedure, see [4], to derive the above mean field vortex density model. The material is in the mixed state where $|\mathbf{w}|>0$ and in the superconducting state where $|\mathbf{w}|=0$. We note that (1.5) gives the velocity of vorticity in samples containing many small pinning sites (cracks and impurities in the material). The given function $f$ is non-decreasing and reflects the resistance to the movement of vorticity by the pinning sites. In $[4,6]$ a linear law was assumed but, as described in [4], one is also interested in more general relations and in particular the situation where the velocity is zero unless the magnitude of the current density exceeds a critical value $J_{p}$, the pinning current.

On the interface between the superconducting sample (which we shall denote by $\Omega$ ) and the external region we have the following boundary conditions;

$$
\begin{aligned}
{\left[\left(1 / \mu_{0}\right) \mathbf{H} \wedge \mathbf{n}\right] } & =\mathbf{0}, \\
{[\mathbf{H} \cdot \mathbf{n}] } & =0,
\end{aligned}
$$

where [.] denotes the jump in the enclosed quantity across $\partial \Omega$ and $\mu_{0}$ is the permeability, which in most practical applications is very similar for the superconducting region and the external region.

The need for an additional boundary condition on $\mathbf{w}$ depends on whether the characteristics of (1.1) are directed into or out of $\Omega$ on the boundary. If $\mathbf{v} \cdot \mathbf{n}>0$, vortices are leaving the sample and no extra boundary conditions are required. However if $\mathbf{v} \cdot \mathbf{n} \leqslant 0$, vortices are moving into the sample and in [4] the following extra boundary condition for the flux of vorticity is suggested;

$$
-\mathbf{n} \wedge(\mathbf{w} \wedge \mathbf{v})=\alpha\left[|\mathbf{J}|-J_{n}\right]_{+} \frac{\mathbf{n} \wedge \mathbf{J}}{|\mathbf{J}|} \quad \text { if } \mathbf{v} \cdot \mathbf{n} \leqslant 0,
$$

where $\alpha$ is a non-negative material constant and $[\cdot]_{+}=\max (\cdot, 0)$. Again there is a critical current density $J_{n}$ below which no vorticity is nucleated.

The model (1.1)-(1.12) can be greatly simplified by considering the case in which all the vortices are rectilinear, aligned and orientated with the $z$-direction along with the magnetic field $\mathbf{H}$. For this 
case we have

$$
\begin{aligned}
& \mathbf{H}=(0,0, u(x, y, t)), \quad u \geqslant 0, \\
& \mathbf{w}=(0,0, w(x, y, t)), \quad w \geqslant 0,
\end{aligned}
$$

and (1.1)-(1.12) simplify to

$$
\begin{gathered}
w_{t}+\nabla \cdot(w \mathbf{v})=0 \quad \text { in } \Omega_{\mathrm{T}}, \\
-\lambda^{2} \Delta u+u=w \quad \text { in } \Omega_{\mathrm{T}}, \\
\mathbf{v}=-\frac{\left[|\nabla u|-J_{p}\right]_{+} \nabla u}{|\nabla u|}, \\
u=u_{\mathrm{b}} \quad \text { on } \partial \Omega_{\mathrm{T}}, \\
-\mathbf{n} \cdot \mathbf{v} w=\alpha\left[|\mathbf{J}|-J_{n}\right]_{+} \quad \text { if } \mathbf{v} \cdot \mathbf{n}<0,
\end{gathered}
$$

where $u_{\mathrm{b}}$ is a positive constant denoting the externally applied magnetic field. We note that in this simplified case, $\operatorname{sgn}(w)=1$ for all $(x, t) \in \Omega_{\mathrm{T}}$ and such situations arise if the initial vorticity $w_{0}(x)$ and the externally applied magnetic field $u_{\mathrm{b}}$ are taken to be non-negative. Restricting our model to one dimension with $\Omega=(0,1)$, we obtain;

$$
\begin{aligned}
& w_{t}+(w v)_{x}=0, \\
& -\lambda^{2} u_{x x}+u=w, \\
& v=-\frac{\left[\left|u_{x}\right|-J_{p}\right]_{+} u_{x}}{\left|u_{x}\right|}=\left\{\begin{array}{cl}
-\left(u_{x}-J_{p}\right) & \text { for } u_{x}>J_{p} \\
0 & \text { for }\left|u_{x}\right| \leqslant J_{p} \\
\left(u_{x}+J_{p}\right) & \text { for } u_{x}<-J_{p}
\end{array}\right. \\
& u(0, t)=u(1, t)=u_{\mathrm{b}}, \\
& v(0, t) w(0, t)=\alpha\left[\left|u_{x}(0, t)\right|-J_{n}\right]_{+} \quad \forall t \in S_{0}=\{t: v(0, t)>0\}, \\
& -v(0, t) w(1, t)=\alpha\left[\left|u_{x}(1, t)\right|-J_{n}\right]_{+} \quad \forall t \in S_{1}=\{t: v(1, t)<0\} .
\end{aligned}
$$

In [9] and [20] the models (1.15)-(1.19) and (1.20)-(1.24) were considered with $J_{p}=0$ and $\alpha=0$, in the remainder of this paper we extend some of the results of [9] and [20] to the following simplified form of (1.20)-(1.24) which is obtained by setting $J_{p}=J_{n}$ and suitably smoothing (1.22);

$$
\begin{array}{cl}
w_{t}=-(w v)_{x} & \text { in } \Omega_{\mathrm{T}}, \\
-\lambda^{2} u_{x x}+u=w & \text { in } \Omega_{\mathrm{T}}, \\
u(0, t)=u(1, t)=u_{\mathrm{b}} & \forall t>0, \\
w(x, 0)=w_{0}(x) & \text { in } \Omega, \\
w(0, t)=w_{\mathrm{b}} & \forall t \in S_{0}=\{t: v(0, t)>0\}, \\
w(1, t)=w_{\mathrm{b}} & \forall t \in S_{1}=\{t: v(1, t)<0\},
\end{array}
$$

where

$$
v=-f\left(u_{x}\right) .
$$


We shall assume that $f \in C^{2}(\mathbb{R})$ satisfies

$$
f(0)=0, \quad 0 \leqslant f^{\prime}(r) \leqslant \mu, \quad\left|f^{\prime \prime}\right| \leqslant C .
$$

We note that (1.29) and (1.30) follow directly from setting $J_{p} \equiv J_{n}$ and $\alpha=w_{\mathrm{b}}$ in (1.22) and (1.24). Also, since the conditions on $f$ in (1.32) are necessary for proving the results of Sections 2-5 we have to content ourselves with a smoother velocity law than the one suggested in [4] which has $f$ being piecewise linear and non-decreasing. In certain applications $\lambda$ is small and it is appropriate to consider the asymptotic limit $\lambda$ tending to zero, which formally leads to a non-linear diffusion equation for the flux line density, cf. $[2,13]$. If in addition to this limit an extreme form of the function $f$ in (1.5) is taken, $f\left(\left|u_{x}\right|\right)=f(|J|)=0$ if $|J| \leqslant J_{p}$ and $f(|J|)=\infty$ if $|J|>J_{p}$, then we formally arrive at the critical-state Bean's model $[18,19]$. A rigorous derivation of Bean's model from a limit of a non-linear diffusion equation may be found in [1]. We note that the vortex density model for an infinite cylinder in a transverse magnetic field has been studied in [10].

In Section 2 we adapt techniques from [20] to prove the existence of a solution $\left\{w^{\varepsilon}, u^{\varepsilon}\right\} \in$ $H^{\beta, \frac{\beta}{2}}\left(\Omega_{\mathrm{T}}\right) \times H^{\beta, \frac{\beta}{2}}\left(\Omega_{\mathrm{T}}\right)$ for $0<\beta<1$, of a regularized version of $(1.25)-(1.30)$ :

$$
\begin{array}{cl}
w_{t}^{\varepsilon}=\left(w^{\varepsilon} f_{\varepsilon}\left(u_{x}^{\varepsilon}\right)\right)_{x}+\varepsilon w_{x x}^{\varepsilon} & \text { in } \Omega_{\mathrm{T}}, \\
-\lambda^{2} u_{x x}^{\varepsilon}+u^{\varepsilon}=w^{\varepsilon} & \text { in } \Omega_{\mathrm{T}}, \\
u^{\varepsilon}(0, t)=u^{\varepsilon}(1, t)=u_{\mathrm{b}} & \forall t>0, \\
w^{\varepsilon}(0, t)=w^{\varepsilon}(1, t)=w_{\mathrm{b}} & \forall t>0, \\
w^{\varepsilon}(x, 0)=w_{0}^{\varepsilon}(x) \geqslant 0 & \text { in } \Omega,
\end{array}
$$

where

$$
f_{\varepsilon}(r)=f(r)+\varepsilon r .
$$

Then we follow the techniques used in [17] to derive estimates on $w^{\varepsilon}, u^{\varepsilon}$ and their derivatives which enable us to prove that in the limit as $\varepsilon \rightarrow 0$

$$
\begin{array}{ll}
w^{\varepsilon} \longrightarrow w & \text { strongly in } L^{1}\left(\Omega_{\mathrm{T}}\right), \\
u^{\varepsilon} \longrightarrow u & \text { strongly in } C\left([0, T] ; C^{1}(\bar{\Omega})\right) .
\end{array}
$$

Here $\{w, u\} \in\left(B V\left(\Omega_{\mathrm{T}}\right) \cap L^{\infty}\left(\Omega_{\mathrm{T}}\right)\right) \times C\left([0, T] ; C^{1}(\bar{\Omega})\right)$ satisfies the following weak form of (1.25)(1.30):

$$
\int_{\Omega_{\mathrm{T}}} w\left(\varphi_{t}-f\left(u_{x}\right) \varphi_{x}\right) \mathrm{d} x \mathrm{~d} t+\int_{\Omega} w_{0}(x) \varphi(x, 0) \mathrm{d} x=0
$$

for all $\varphi \in C^{\infty}\left(\Omega_{\mathrm{T}}\right),\left.\varphi\right|_{(0,1) \times\{T\} \cup \partial \Omega \times(0, T)}=0$,

$$
\int_{\Omega} \lambda^{2} u_{x} \phi_{x} \mathrm{~d} x+\int_{\Omega}(u-w) \phi \mathrm{d} x=0 \quad \forall \phi \in C\left([0, T] ; H_{0}^{1}(\Omega)\right),
$$

and the entropy inequality

$$
\begin{aligned}
\int_{\Omega_{\mathrm{T}}}(-(w- & \left.\left.w_{\mathrm{b}}\right)^{2} \eta_{t}+\left(w-w_{\mathrm{b}}\right)^{2} f\left(u_{x}\right) \eta_{x}\right) \mathrm{d} x \\
& \leqslant \int_{\Omega_{\mathrm{T}}}\left(\left(\left(w-w_{\mathrm{b}}\right)^{2}-2 w\left(w-w_{\mathrm{b}}\right)\right) \lambda^{-2} f^{\prime}\left(u_{x}\right)(w-u) \eta\right) \mathrm{d} x \mathrm{~d} t
\end{aligned}
$$


where $\eta \in W^{1, \infty}\left(\Omega_{\mathrm{T}}\right)$ with $\eta(\cdot, 0)=\eta(\cdot, T)=0$ and $\eta \geqslant 0$. Since $B V$ functions have traces on the boundary, we are able to use the entropy inequality (1.41) to prove that $w$ satisfies the boundary conditions (1.29) and (1.30). In Section 5 we prove the existence of a Lyapunov functional

$$
E(u(\cdot, t))=\frac{1}{2} \int_{\Omega}\left(\left|u_{x}\right|^{2}+\left(u-u_{\mathrm{b}}\right)^{2}\right) \mathrm{d} x,
$$

from which we conclude the existence of a steady-state solution $\{\tilde{w}, \tilde{u}\} \in B V(\Omega) \times W^{2, \infty}(\Omega)$ which satisfies

$$
\begin{array}{cl}
\tilde{w} f\left(\tilde{u}_{x}\right)=0 & \text { in } \Omega, \\
-\lambda^{2} \tilde{u}_{x x}+\tilde{u}=\tilde{w} \geqslant 0 & \text { in } \Omega, \\
\tilde{u}(0)=\tilde{u}(1)=u_{\mathrm{b}} & \text { on } \partial \Omega, \\
\tilde{w}(0)=w_{\mathrm{b}} & \text { if } f\left(\tilde{u}_{x}(0)\right)<0, \\
\tilde{w}(1)=w_{\mathrm{b}} & \text { if } f\left(\tilde{u}_{x}(1)\right)>0 .
\end{array}
$$

In Section 6 we adapt techniques from [9] and [8] to derive a numerical discretization of $(1.25)-(1.30)$ on the interval $[0,1]$ using a uniform mesh size $h$. We show that as the time step and the mesh size tend to zero, the discrete solution converges to the unique weak solution of the continuous model. In Section 7 we show that as $t \rightarrow \infty$ there exists a unique sequence $\left\{\tilde{w}_{h}, \tilde{u}_{h}\right\} \in L^{\infty}(\Omega) \times H^{1}(\Omega)$ which satisfies a discrete form of the continuous steady-state problem. We conclude with Section 8 in which we display some one- and two-dimensional numerical computations that illustrate the effects of flux pinning and boundary nucleation coupled with a finite penetration depth. We note that in order to keep the paper from being too long, some of the proofs in the following sections have been shortened, the full versions of these proofs can be found in the technical report [11].

\section{Existence of a solution of the regularized model}

THEOREM 2.1 For all initial data $w_{0}^{\varepsilon}(\cdot)-w_{\mathrm{b}} \in C_{0}^{\infty}(\Omega)$, there exists a classical solution $\left\{w^{\varepsilon}, u^{\varepsilon}\right\}$ of (1.33)-(1.37) with

$$
\left\|w^{\varepsilon}\right\|_{H^{\alpha, \frac{\alpha}{2}}\left(\Omega_{\mathrm{T}}\right)}, \quad\left\|u^{\varepsilon}\right\|_{H^{\alpha, \frac{\alpha}{2}\left(\Omega_{\mathrm{T}}\right)}}, \quad\left\|u_{x}^{\varepsilon}\right\|_{H^{\alpha, \frac{\alpha}{2}\left(\Omega_{\mathrm{T}}\right)}} \leqslant C,
$$

for $0<\alpha<1$.

Proof. Throughout this proof, for the sake of simplicity of presentation, we often suppress the dependence of $u^{\varepsilon}$ and $w^{\varepsilon}$ on $\varepsilon$ and write $w$ and $u$ in place of $w^{\varepsilon}$ and $u^{\varepsilon}$. We begin by embedding (1.33)-(1.37) in the following family of problems $P^{\sigma}$ : find $v^{\sigma} \in H^{2+\alpha, 1+\frac{\alpha}{2}}\left(\Omega_{\mathrm{T}}\right)$ such that

$$
\begin{array}{cc}
v_{t}^{\sigma}-\varepsilon v_{x x}^{\sigma}-v_{x}^{\sigma} f_{\varepsilon}\left(u_{x}\right)-\sigma^{2} \lambda^{-2} f_{\varepsilon}^{\prime}\left(u_{x}\right) v^{\sigma} u+\sigma \lambda^{-2} f_{\varepsilon}^{\prime}\left(u_{x}\right) w^{2}=0 & \text { in } \Omega_{\mathrm{T}}, \\
v^{\sigma}(0, t)=v^{\sigma}(1, t)=\sigma w_{\mathrm{b}}, & \text { in } \Omega, \\
v^{\sigma}(\cdot, 0)-w_{\mathrm{b}}=\sigma w_{0}(x)-w_{\mathrm{b}} \in C_{0}^{\infty}(\Omega) & \text { in }
\end{array}
$$

where

$$
w \in H^{\alpha, \frac{\alpha}{2}}\left(\Omega_{\mathrm{T}}\right)
$$


and $u$ satisfies

$$
\begin{array}{r}
-\lambda^{2} u_{x x}+u=w \quad \text { in } \Omega_{\mathrm{T}}, \\
u(0, t)=u(1, t)=u_{\mathrm{b}} .
\end{array}
$$

From [16] we see that if the coefficients of $v^{\sigma}$ in (2.2) are bounded in $H^{\alpha, \frac{\alpha}{2}}\left(\Omega_{\mathrm{T}}\right)$, (i.e. if $f_{\varepsilon}\left(u_{x}\right)$, $\sigma^{2} \lambda^{-2} f_{\varepsilon}^{\prime}\left(u_{x}\right) u$ and $\sigma \lambda^{-2} f_{\varepsilon}^{\prime}\left(u_{x}\right) w^{2}$ are bounded in $\left.H^{\alpha, \frac{\alpha}{2}}\left(\Omega_{\mathrm{T}}\right)\right)$, and if $v^{\sigma}(\cdot, 0) \in C^{2, \alpha}(\Omega)$ and $\sigma w_{\mathrm{b}} \in H^{2+\alpha, 1+\frac{\alpha}{2}}\left(\partial \Omega_{\mathrm{T}}\right)$, there exists a unique solution $v^{\sigma} \in H^{2+\alpha, 1+\frac{\alpha}{2}}\left(\Omega_{\mathrm{T}}\right)$ of $P^{\sigma}$. Since $w \in$ $H^{\alpha, \frac{\alpha}{2}}\left(\Omega_{\mathrm{T}}\right)$ from (2.4), we have

$$
\begin{aligned}
& w(\cdot, t) \in C^{0, \alpha}(\Omega) \Rightarrow u(\cdot, t) \in C^{2, \alpha}(\Omega) \quad \forall t>0 \\
& \Rightarrow\left\|u\left(\cdot, t_{2}\right)-u\left(\cdot, t_{1}\right)\right\|_{C^{1, \alpha}(\Omega)} \leqslant C\left\|u\left(\cdot, t_{2}\right)-u\left(\cdot, t_{1}\right)\right\|_{W^{2, \infty}(\Omega)} \\
& \leqslant C\left\|w\left(\cdot, t_{2}\right)-w\left(\cdot, t_{1}\right)\right\|_{L^{\infty}(\Omega)} \\
& \leqslant C\left|t_{2}-t_{1}\right|^{\frac{\alpha}{2}} \\
& \left.\Rightarrow\|u\|\right|_{H^{\alpha, \frac{\alpha}{2}}\left(\Omega_{\mathrm{T}}\right)}, \quad\left\|u_{x}\right\|_{H^{\alpha, \frac{\alpha}{2}}\left(\Omega_{\mathrm{T}}\right)} \leqslant C .
\end{aligned}
$$

From (1.32) and (2.6) it follows that

$$
\left\|f_{\varepsilon}\left(u_{x}\right)\right\|_{H^{\alpha, \frac{\alpha}{2}\left(\Omega_{\mathrm{T}}\right)}} \leqslant C,
$$

and hence there exists a unique solution $v^{\sigma} \in H^{2+\alpha, 1+\frac{\alpha}{2}}\left(\Omega_{\mathrm{T}}\right)$ of $P^{\sigma}$. Setting $v^{\sigma}=T(w, \sigma)$ for all $w \in H^{\alpha, \frac{\alpha}{2}}\left(\Omega_{\mathrm{T}}\right)$ and all $\sigma \in[0,1]$, and applying the Leray-Schauder fixed point theorem [12], we see that solving (1.33)-(1.37) in $H^{2+\alpha, 1+\frac{\alpha}{2}}\left(\Omega_{\mathrm{T}}\right)$ is equivalent to solving $w=T(w, 1)$ in $H^{\alpha, \frac{\alpha}{2}}\left(\Omega_{\mathrm{T}}\right)$. In order to apply the Leray-Schauder fixed point theorem we need to prove that the mapping $T$ is continuous and compact and that

$$
\begin{gathered}
T(w, 0)=0 \quad \forall w \in H^{\alpha, \frac{\alpha}{2}}\left(\Omega_{\mathrm{T}}\right), \\
\|w=T(w, \sigma)\|_{H^{\alpha, \frac{\alpha}{2}\left(\Omega_{\mathrm{T}}\right)}}<M \quad \forall(w, \sigma) \in H^{\alpha, \frac{\alpha}{2}}\left(\Omega_{\mathrm{T}}\right) \times[0,1] .
\end{gathered}
$$

The compactness of the mapping $T$ follows from the fact that $T$ maps bounded sets in $H^{\alpha, \frac{\alpha}{2}}\left(\Omega_{\mathrm{T}}\right) \times$ $[0,1]$ into bounded sets in $H^{2+\alpha, 1+\frac{\alpha}{2}}\left(\Omega_{\mathrm{T}}\right)$, which are compactly embedded in $H^{\alpha, \frac{\alpha}{2}}\left(\Omega_{\mathrm{T}}\right)$, [16]. Now we prove the continuity of $T$. Using (1.34) and (2.6) we conclude that there exists subsequences $w_{j}$ and $u_{j}$ such that

$$
\begin{gathered}
\left\|u_{j}(\cdot, t)-u(\cdot, t)\right\|_{C^{2, \alpha}(\Omega)} \leqslant C\left\|w_{j}(\cdot, t)-w(\cdot, t)\right\|_{C^{0, \alpha}(\Omega)} \rightarrow 0 \\
\left.\Rightarrow \begin{array}{l}
u_{j} \rightarrow u \\
\left(u_{x}\right)_{j} \rightarrow u_{x}
\end{array}\right\} \text { in } H^{\beta, \frac{\beta}{2}}\left(\Omega_{\mathrm{T}}\right) \quad \text { for } 0<\beta<\alpha .
\end{gathered}
$$

Using (1.32) and (2.10) it follows that

$$
\left.\Rightarrow \begin{array}{l}
f_{\varepsilon}\left(\left(u_{x}\right)_{j}\right) \rightarrow f_{\varepsilon}\left(u_{x}\right) \\
f_{\varepsilon}^{\prime}\left(\left(u_{x}\right)_{j}\right) \rightarrow f_{\varepsilon}^{\prime}\left(u_{x}\right)
\end{array}\right\} \text { in } H^{\beta, \frac{\beta}{2}}\left(\Omega_{\mathrm{T}}\right) \quad \text { for } 0<\beta<\alpha .
$$


Also $\sigma w_{0}(x)-w_{\mathrm{b}} \in C_{0}^{\infty}(\Omega), \sigma w^{2} \in H^{\alpha, \frac{\alpha}{2}}\left(\Omega_{\mathrm{T}}\right)$ imply

$$
\begin{array}{ll}
\sigma_{j} w_{0} \rightarrow \sigma w_{0} & \text { in } C^{2, \beta}(\Omega), \\
\sigma_{j} w_{j}^{2} \rightarrow \sigma w^{2} & \text { in } H^{\beta, \frac{\beta}{2}}\left(\Omega_{\mathrm{T}}\right) .
\end{array}
$$

Since $H^{2+\alpha, 1+\frac{\alpha}{2}}\left(\Omega_{\mathrm{T}}\right)$ is compactly embedded in $H^{\alpha, \frac{\alpha}{2}}\left(\Omega_{\mathrm{T}}\right)$ every subsequence $v_{j}^{\sigma_{j}}$ of $v^{\sigma}$ has a convergent subsequence $v_{j_{k}}^{\sigma_{j_{k}}}$. Let $v_{j}^{\sigma_{j}}$ be such a convergent subsequence with limit $\bar{v}^{\sigma} \in H^{\alpha, \frac{\alpha}{2}}\left(\Omega_{\mathrm{T}}\right)$. Then using (2.11), (2.12) and (2.13) we have

$$
\begin{gathered}
\lim _{j \rightarrow \infty} v_{j t}^{\sigma_{j}}-\varepsilon v_{j x x}^{\sigma_{j}}-v_{j x}^{\sigma_{j}} f_{\varepsilon}\left(\left(u_{x}\right)_{j}\right)-\sigma_{j}^{2} \lambda^{-2} f_{\varepsilon}^{\prime}\left(u_{j x}\right) v_{j}^{\sigma_{j}} u+\sigma_{j} \lambda^{-2} f_{\varepsilon}^{\prime}\left(u_{x}\right)_{j} w_{j}^{2} \\
=\bar{v}_{t}^{\sigma}-\varepsilon \bar{v}_{x x}^{\sigma}-\bar{v}_{x}^{\sigma} f_{\varepsilon}\left(u_{x}\right)-\sigma_{j}^{2} \lambda^{-2} f_{\varepsilon}^{\prime}\left(u_{x}\right) \bar{v}^{\sigma} u+\sigma_{j} \lambda^{-2} f_{\varepsilon}^{\prime}\left(u_{x}\right) \sigma w^{2}=0 \quad \text { in } \Omega_{\mathrm{T}}, \\
\bar{v}^{\sigma}(0, t)=\bar{v}^{\sigma}(1, t)=\sigma w_{\mathrm{b}}, \\
\bar{v}^{\sigma}(\cdot, 0)=\sigma w_{0}(x) \text { in } \Omega .
\end{gathered}
$$

Since $P^{\sigma}$ admits a unique solution we conclude the sequence $v_{j}^{\sigma_{j}}$ itself converges to $\bar{v}^{\sigma}$ and the continuity of the mapping is proved. To complete the proof we first note that if $\sigma=0$, then $w=0$ and hence it remains to set $w=T(w, \sigma)$ and prove (2.9). Following the techniques used in the proof of Theorem 2.1 [20], and noting that $f_{\varepsilon}^{\prime}>0$ we arrive at the following

$$
\begin{gathered}
0=\min \left(w_{0}(x), u_{\mathrm{b}}, w_{\mathrm{b}}\right) \leqslant \min w^{\sigma} \leqslant \max w \leqslant \max \left(w_{0}(x), u_{\mathrm{b}}, w_{\mathrm{b}}\right), \\
\left\|w^{\sigma}\right\|_{H^{2+\alpha, 1+\frac{\alpha}{2}}\left(\Omega_{\mathrm{T}}\right)} \leqslant C \quad \forall \sigma \in[0,1),
\end{gathered}
$$

where $w^{\sigma}=T\left(w^{\sigma}, \sigma\right)$ and $0<\sigma<1$. Hence there exists a subsequence of $w^{\sigma}$, which for simplicity we denote $w^{\sigma}$, such that

$$
\begin{gathered}
w^{\sigma} \longrightarrow w \quad \text { in } H^{2+\beta, 1+\frac{\beta}{2}}\left(\Omega_{\mathrm{T}}\right) \text { as } \sigma \rightarrow 1, \\
\Rightarrow w \longleftarrow w^{\sigma}=T\left(w^{\sigma}, \sigma\right) \longrightarrow T(w, 1),
\end{gathered}
$$

i.e.

$$
\|w\|_{H^{\alpha, \frac{\alpha}{2}}\left(\Omega_{\mathrm{T}}\right)} \leqslant C \quad \forall \sigma \in[0,1]
$$

and the theorem is proved.

LEMma 2.1 The solution $w^{\varepsilon}$ of (1.33)-(1.37) satisfies

$$
\begin{gathered}
0 \leqslant w^{\varepsilon}(x, t) \leqslant \max \left(w_{0}^{\varepsilon}(x), u_{\mathrm{b}}, w_{\mathrm{b}}\right) \leqslant C . \\
\left\|u^{\varepsilon}\right\|_{L^{\infty}\left(0, T ; W^{2, \infty}(\Omega)\right.} \leqslant C .
\end{gathered}
$$

Proof. The result follows directly from the proof of Theorem 2.1. 


\section{Existence of a weak solution of the model}

Throughout this section, for the sake of simplicity of presentation, we often suppress the dependence of $u^{\varepsilon}$ and $w^{\varepsilon}$ on $\varepsilon$ and write $w$ and $u$ in place of $w^{\varepsilon}$ and $u^{\varepsilon}$.

We begin by choosing regularized initial data $w_{0}^{\varepsilon}(x)-w_{\mathrm{b}} \in C_{0}^{\infty}(\Omega)$, such that

$$
w_{0}^{\varepsilon}(x) \longrightarrow w_{0}(x) \text { strongly in } L^{p}(\Omega) \quad \forall 1 \leqslant p<\infty,
$$

and

$$
\left\|w_{0}^{\varepsilon}\right\|_{W^{1,1}(\Omega)} \leqslant C, \quad\left\|\varepsilon w_{0}^{\varepsilon}\right\|_{W^{2,1}(\Omega)} \leqslant C .
$$

LEMMA 3.1 There exist constants $C$ independent of $\varepsilon$, such that

$$
\begin{aligned}
& \left\|\sqrt{\varepsilon} w_{x}^{\varepsilon}\right\|_{L^{2}\left(\Omega_{\mathrm{T}}\right)} \leqslant C, \\
& \left\|u_{t}^{\varepsilon}\right\|_{L^{2}\left(0, T ; H_{0}^{1}(\Omega)\right)} \leqslant C . \\
& \left\|u^{\varepsilon}\right\|_{H^{\beta, \beta / 2}\left(\Omega_{\mathrm{T}}\right)}, \quad\left\|u_{x}^{\varepsilon}\right\|_{H^{\beta, \beta / 2}\left(\Omega_{\mathrm{T}}\right)} \leqslant C,
\end{aligned}
$$

for some $0<\beta<1$.

We omit the proof of Lemma 3.1 since it follows the same techniques used in the proof of Lemma 3.1 [20] and can be found in [11].

LEMmA 3.2 There exists a $C$ independent of $\varepsilon$, such that

$$
\left\|w_{x}^{\varepsilon}\right\|_{L^{\infty}\left(0, T ; L^{1}(0,1)\right)} \leqslant C .
$$

Proof. Differentiating (1.33) with respect to $x$, multiplying the resulting equation by $\operatorname{sgn}\left(w_{x}\right)$ and integrating from $x=0$ to $x=1$ gives

$$
\begin{aligned}
\frac{\mathrm{d}}{\mathrm{d} t} \int_{0}^{1}\left|w_{x}\right| \mathrm{d} x= & \varepsilon \int_{0}^{1} w_{x x x} \operatorname{sgn}\left(w_{x}\right) \mathrm{d} x+2 \int_{0}^{1} f_{\varepsilon}^{\prime}\left(u_{x}\right) u_{x x}\left|w_{x}\right| \mathrm{d} x \\
& +\int_{0}^{1}\left(w_{x x} f_{\varepsilon}\left(u_{x}\right)+w f_{\varepsilon}^{\prime}\left(u_{x}\right) u_{x x x}\right) \operatorname{sgn}\left(w_{x}\right) \mathrm{d} x \\
= & \varepsilon \int_{0}^{1} w_{x x x} \operatorname{sgn}\left(w_{x}\right) \mathrm{d} x+2 \int_{0}^{1} f_{\varepsilon}^{\prime}\left(u_{x}\right) u_{x x}\left|w_{x}\right| \mathrm{d} x \\
& +\int_{0}^{1} f_{\varepsilon}\left(u_{x}\right)\left|w_{x}\right|_{x} \mathrm{~d} x+\int_{0}^{1} f_{\varepsilon}^{\prime}\left(u_{x}\right) w u_{x x x} \operatorname{sgn}\left(w_{x}\right) \mathrm{d} x \\
& +\int_{0}^{1} w f_{\varepsilon}^{\prime \prime}\left(u_{x}\right)\left(u_{x x}\right)^{2} \operatorname{sgn}\left(w_{x}\right) \mathrm{d} x .
\end{aligned}
$$

Following the techniques used in the proof of Lemma 5.1 [20], which in particular involve using (1.34) coupled with the boundedness of $u_{x}$ to deal with the term involving the third derivative of $u$, using the following useful inequality

$$
\varepsilon \int_{0}^{1} w_{x x x} \operatorname{sgn}\left(w_{x}\right) \mathrm{d} x+\left[\left|w_{x}\right| u_{x}\right]_{0}^{1} \leqslant 0
$$


to deal with the $\varepsilon \int_{0}^{1} w_{x x x} \mathrm{~d} x$ term, and finally noting from (1.32) that $f_{\varepsilon}^{\prime}\left(u_{x}\right)$ and $f_{\varepsilon}^{\prime \prime}\left(u_{x}\right)$ are bounded in $L^{\infty}\left(\Omega_{\mathrm{T}}\right)$ we arrive at the following

$$
\begin{aligned}
\frac{\mathrm{d}}{\mathrm{d} t} \int_{0}^{1}\left|w_{x}\right| \mathrm{d} x \leqslant & \int_{0}^{1}\left(\left|w_{x}\right| f_{\varepsilon}^{\prime}\left(u_{x}\right) u_{x x}+w f_{\varepsilon}^{\prime}\left(u_{x}\right)(u-w)_{x}\right) \operatorname{sgn}\left(w_{x}\right) \mathrm{d} x \\
& +\int_{0}^{1} \lambda^{-2} f_{\varepsilon}^{\prime \prime}\left(u_{x}\right) w(w-u)^{2} \operatorname{sgn}\left(w_{x}\right) \mathrm{d} x \\
\leqslant & C \int_{0}^{1}\left|w_{x}\right| \mathrm{d} x+C \int_{0}^{1}\left|u_{x}\right| \mathrm{d} x+C .
\end{aligned}
$$

Using (3.5) and Gronwall's inequality in (3.8) gives the required result.

Lemma 3.3 There exists a constant $C$ independent of $\varepsilon$, such that

$$
\left\|w_{t}^{\varepsilon}\right\|_{L^{\infty}\left(0, T ; L^{1}(0,1)\right)} \leqslant C \text {. }
$$

The proof of Lemma 3.3 is essentially the same as the proof of Lemma 5.3 [20], with the exception of some terms involving $f_{\varepsilon}^{\prime}(\cdot)$ and $f_{\varepsilon}^{\prime \prime}(\cdot)$ which can be bounded in $L^{\infty}\left(\Omega_{\mathrm{T}}\right)$.

THEOREM 3.1 For every sequence $\left\{w^{\varepsilon}, u^{\varepsilon}\right\}$, of solutions of (1.33)-(1.37), there exists a subsequence, which for simplicity of notation we also denote by $\left\{w^{\varepsilon}, u^{\varepsilon}\right\}$, which converges as $\varepsilon \rightarrow 0$, in $L^{1}\left(\Omega_{\mathrm{T}}\right) \times C\left(\overline{\Omega_{\mathrm{T}}}\right)$ to $\{w, u\}$. We obtain

$$
\begin{gathered}
w \in L^{\infty}(0, T ; B V(\Omega)) \quad \text { and } \quad w \in L^{\infty}\left(\Omega_{\mathrm{T}}\right) \cap B V\left(\Omega_{\mathrm{T}}\right), \\
u, u_{x} \in H^{\beta, \beta / 2}\left(\Omega_{\mathrm{T}}\right) \subseteq C\left(\overline{\Omega_{\mathrm{T}}}\right) \quad \text { for some } 0<\beta<1 .
\end{gathered}
$$

Moreover, $\{w, u\}$ is a weak solution of (1.39) and (1.40), and it satisfies the entropy inequality (1.41).

Proof. We begin by noting that (3.10) and (3.11) are direct consequences of (2.15), (3.5), (3.6) and (3.9). Furthermore, the following convergence results follow from (1.32), (1.38) and (3.5),

$$
\begin{array}{cl}
u^{\varepsilon} \rightarrow u & \text { strongly in } C\left(\overline{\Omega_{\mathrm{T}}}\right), \\
u_{x}^{\varepsilon} \rightarrow u_{x} & \text { strongly in } C\left(\overline{\Omega_{\mathrm{T}}}\right), \\
f_{\varepsilon}\left(u_{x}^{\varepsilon}\right) \rightarrow f\left(u_{x}\right) & \text { strongly in } C\left([0, T] ; L^{2}(\Omega)\right), \\
f_{\varepsilon}^{\prime}\left(u_{x}^{\varepsilon}\right) \rightarrow f^{\prime}\left(u_{x}\right) & \text { strongly in } C\left([0, T] ; L^{2}(\Omega)\right) .
\end{array}
$$

Lastly, from (3.6) and (3.9) it follows that $w^{\varepsilon} \in W^{1,1}\left(\Omega_{\mathrm{T}}\right)$ and since $L^{1}$ is compactly embedded in $W^{1,1}$ we conclude

$$
w^{\varepsilon} \longrightarrow w \text { strongly in } L^{1}\left(\Omega_{\mathrm{T}}\right) .
$$

To prove that $\{w, u\}$ satisfies (1.39) we multiply (1.33) by $\varphi \in C^{\infty}\left(\Omega_{\mathrm{T}}\right)$ where supp $\varphi \subseteq \Omega \times\{T\} \cup$ $\partial \Omega_{\mathrm{T}}$ and integrate over $\Omega_{\mathrm{T}}$, use the convergence properties (3.12)-(3.15) and follow the techniques used in the proof of Theorem 3.1 in [20], to obtain

$$
\begin{array}{r}
\int_{\Omega_{\mathrm{T}}} w^{\varepsilon}\left(\varphi_{t}-f_{\varepsilon}\left(u_{x}^{\varepsilon}\right) \varphi_{x}\right) \mathrm{d} x \mathrm{~d} t+\int_{\Omega} w_{0}^{\varepsilon} \varphi(x, 0) \mathrm{d} x+\varepsilon \int_{\Omega_{\mathrm{T}}} w^{\varepsilon} \varphi_{x x} \mathrm{~d} x \mathrm{~d} t \\
\longrightarrow \int_{\Omega_{\mathrm{T}}} w\left(\varphi_{t}-f\left(u_{x}\right) \varphi_{x}\right) \mathrm{d} x \mathrm{~d} t+\int_{\Omega} w_{0} \varphi(x, 0) \mathrm{d} x .
\end{array}
$$


Similarly, multiplying (1.34) by $\phi \in C\left([0, T]: H^{1}(\Omega)\right)$, integrating over $\Omega$, using the convergence properties (3.12)-(3.15) and following the techniques used in the proof of Theorem 3.1 in [20] we obtain

$$
\int_{\Omega}\left(\lambda^{2} u_{x}^{\varepsilon} \phi_{x}-\left(w^{\varepsilon}-u^{\varepsilon}\right) \phi\right) \mathrm{d} x \rightarrow \int_{\Omega}\left(\lambda^{2} u_{x} \phi_{x}-(w-u) \phi\right) \mathrm{d} x .
$$

It remains to prove that $\{w, u\}$ satisfies (1.41). To this end, we multiply (1.33) by $w^{\varepsilon}-w_{\mathrm{b}}$ to obtain

$$
\begin{gathered}
\left(w^{\varepsilon}-w_{\mathrm{b}}\right) w_{t}^{\varepsilon}-\left(w^{\varepsilon}-w_{\mathrm{b}}\right)\left(w^{\varepsilon} f_{\varepsilon}\left(u_{x}^{\varepsilon}\right)\right)_{x}-\varepsilon\left(w^{\varepsilon}-w_{\mathrm{b}}\right) w_{x x}^{\varepsilon}=0 \\
\Rightarrow \frac{\mathrm{d}}{\mathrm{d} t}\left(w^{\varepsilon}-w_{\mathrm{b}}\right)^{2}-\left(w^{\varepsilon}-w_{\mathrm{b}}\right)_{x}^{2} f_{\varepsilon}\left(u_{x}^{\varepsilon}\right)+2 \lambda^{-2} f_{\varepsilon}^{\prime}\left(u_{x}\right) w^{\varepsilon}\left(w^{\varepsilon}-w_{\mathrm{b}}\right)\left(w^{\varepsilon}-u^{\varepsilon}\right)-\varepsilon\left(w^{\varepsilon}-w_{\mathrm{b}}\right)_{x x}^{2} \leqslant 0 .
\end{gathered}
$$

Multiplying the above inequality by $\eta \in W^{1, \infty}\left(\Omega_{\mathrm{T}}\right), \eta(0)=\eta(T)=0$, and integrating over $\Omega_{\mathrm{T}}$ gives

$$
\begin{gathered}
\int_{\Omega_{\mathrm{T}}}\left(-\left(w^{\varepsilon}-w_{\mathrm{b}}\right)^{2} \eta_{t}+\left(w^{\varepsilon}-w_{\mathrm{b}}\right)^{2} f_{\varepsilon}\left(u_{x}^{\varepsilon}\right) \eta_{x}+\lambda^{-2} f_{\varepsilon}^{\prime}\left(u_{x}\right)\left(w^{\varepsilon}-u^{\varepsilon}\right)\left(2 w^{\varepsilon}\left(w^{\varepsilon}-w_{\mathrm{b}}\right) \eta\right) \mathrm{d} x \mathrm{~d} t\right. \\
\left.-\int_{\Omega_{\mathrm{T}}}\left(\lambda^{-2} f_{\varepsilon}^{\prime}\left(u_{x}\right)\left(w^{\varepsilon}-u^{\varepsilon}\right)\left(w^{\varepsilon}-w_{\mathrm{b}}\right)^{2}\right) \eta\right) \mathrm{d} x \mathrm{~d} t \leqslant-\int_{\Omega_{\mathrm{T}}} \varepsilon\left(w^{\varepsilon}-w_{\mathrm{b}}\right)_{x}^{2} \eta_{x} \mathrm{~d} x \mathrm{~d} t .
\end{gathered}
$$

Using (2.15) and (3.3) it follows that in the limit as $\varepsilon \rightarrow 0$ the right-hand side of the above inequality converges to zero, while from (3.12), (3.13) and (3.16) we can conclude that in the limit as $\varepsilon \rightarrow 0$ the left-hand side of the above inequality converges to (1.41) and the theorem is proved.

LEMmA 3.4 The limiting solution $\{w, u\}$ satisfies the boundary conditions

$$
\begin{array}{cc}
u(0, t)=u(1, t)=u_{\mathrm{b}} & \forall t>0, \\
w(0, t)=w_{\mathrm{b}} & \forall t \in S_{0}, \\
w(1, t)=w_{\mathrm{b}} & \forall t \in S_{1},
\end{array}
$$

where the sets $S_{0}$ and $S_{1}$ are defined by

$$
S_{0}=\left\{\tau>0: f\left(u_{x}(0, \tau)\right)<0\right\} \quad \text { and } \quad S_{1}=\left\{\tau>0: f\left(u_{x}(1, \tau)\right)>0\right\} .
$$

We omit the proof of Lemma 3.4 as it is a straightforward generalization of part of the proof of Theorem 5.1 in [20].

\section{Uniqueness of the weak solution}

In Section 3 we proved the existence of a weak solution satisfying (1.27), (1.29), (1.30), (1.39), (1.40) and (1.41). If this weak solution is unique (which we prove in this section), Theorem 3.1 implies the convergence of solutions of (1.33)-(1.37) to the weak solution of (1.25)-(1.30), i.e.

$$
\lim _{\varepsilon \rightarrow 0}\left\{w^{\varepsilon}, u^{\varepsilon}\right\}=\{w, u\}
$$

We now prove the uniqueness of weak solutions of the limit problem (1.25)-(1.30). The main difficulty in the proof is caused by the fact that the weak solution $w$ is not necessarily smooth, 
so we bypass this difficulty by smoothing it appropriately. In order to do this we firstly extend $u_{x}$ to $\mathbb{R} \times[0, T]$ by

$$
u_{x}(x, t)= \begin{cases}u_{x}(0, t) & x<0, \\ u_{x}(x, t) & 0 \leqslant x \leqslant 1, \\ u_{x}(1, t) & x>1,\end{cases}
$$

and we define the 'extended' characteristic $X=X(t ; \bar{x}, \bar{t})$ to be the unique solution of the initial value problem

$$
\begin{gathered}
X_{t}=-f\left(u_{x}(X, t)\right), \quad t \in \mathbb{R}, \\
X(\bar{t} ; \bar{x}, \bar{t})=\bar{x} \in \mathbb{R} .
\end{gathered}
$$

We note that $f\left(u_{x}\right) \in L^{\infty}\left(0, T ; C^{0,1}(\Omega)\right) \cap C\left([0, T] ; C^{0, \alpha}(\bar{\Omega})\right) 0 \leqslant \alpha<1$, for all $t \geqslant 0$, and using the Picard-Lindelof Theorem [15], it follows that (4.1)-(4.2) has a unique solution. Next we set up the coordinate transformation $(x, t) \leftrightarrow(s, \tau)$ to be the following,

$$
(s, \tau) \leftrightarrow(x, t) \quad\left\{\begin{array}{l}
s=X(0 ; x, t), \\
\tau=t,
\end{array}\right.
$$

(i.e. $s$ is the initial value of the 'extended' characteristic $X$, which passes through $(x, t)$ ), and we note that from (4.3) it follows that

$$
x=X(\tau ; s, 0) .
$$

Lastly, we define $Q_{\mathrm{T}}$ to be the image of $\Omega_{\mathrm{T}}$ in the $(s, \tau)$-plane, we set $\tilde{w}(s, \tau)=w(x, t)$ and extend $\tilde{w}$ to $s \in \mathbb{R}$ by its boundary values.

LEMMA 4.1 The function $\tilde{w}$ has a bounded $\tau$ derivative:

$$
\tilde{w}_{\tau}=\tilde{w} f^{\prime}\left(u_{x}\right) u_{x x} \in L^{\infty}\left(Q_{\mathrm{T}}\right) .
$$

Proof. Using the extended value of $u_{x}$ and the coordinate transformation $(s, t) \leftrightarrow(x, t)$ defined above, we have

$$
\operatorname{det}\left(\frac{\partial(s, \tau)}{\partial(x, t)}\right)=\left|\begin{array}{cc}
\frac{\partial s}{\partial x} & \frac{\partial s}{\partial t} \\
\frac{\partial \tau}{\partial x} & \frac{\partial \tau}{\partial t}
\end{array}\right|=\frac{\partial X(0 ; x, t)}{\partial x} .
$$

Since

$$
\frac{\partial}{\partial t} \frac{\partial X}{\partial x}=-f^{\prime}\left(u_{x}\right) u_{x x}(X, t) \frac{\partial X}{\partial x},
$$

with

$$
\frac{\partial X}{\partial x}(0 ; x, 0)=1,
$$

we obtain

$$
\begin{gathered}
\frac{\partial X(0 ; x, t)}{\partial x}=\exp \left[\int_{0}^{t} f^{\prime}\left(u_{x}\right) u_{x x}(X, r) \mathrm{d} r\right] \\
\Rightarrow \operatorname{det}\left(\frac{\partial(x, t)}{\partial(s, \tau)}\right)=\exp \left[-\int_{0}^{t} f^{\prime}\left(u_{x}\right) u_{x x}(X, r) \mathrm{d} r\right] \neq 0 .
\end{gathered}
$$


Since $\operatorname{det}\left(\frac{\partial(x, t)}{\partial(s, \tau)}\right) \neq 0$, it follows that the coordinate transformation $(x, t) \leftrightarrow(s, \tau)$ is invertible and using standard arguments it can be shown that $(x, t) \leftrightarrow(s, \tau)$ and its inverse $(s, \tau) \leftrightarrow(x, t)$ are in $C_{\text {loc }}^{0,1}(\mathbb{R} \times[0, T])$. For $\varphi \in C_{0}^{\infty}\left(\Omega_{\mathrm{T}}\right)$ the weak formulation of (1.39) reads

$$
\int_{\Omega_{\mathrm{T}}} w\left(\varphi_{t}-f\left(u_{x}\right) \varphi_{x}\right) \mathrm{d} x \mathrm{~d} t=0
$$

We now set $\tilde{\varphi}(s, \tau)=\varphi(x, t)$ and from (4.4) we have $\tilde{\varphi}_{\tau}=\varphi_{t}-f\left(u_{x}\right) \varphi_{x}$, which together with the change of variables $s=s(x, t), \tau=t$ gives

$$
\int_{Q_{\mathrm{T}}} \tilde{w} \tilde{\varphi}_{\tau}\left|\frac{\partial(x, t)}{\partial(s, \tau)}\right| \mathrm{d} s \mathrm{~d} \tau=0 .
$$

Thus

$$
\int_{Q_{\mathrm{T}}} \tilde{w} \exp \left[-\int_{0}^{\tau} f^{\prime}\left(u_{x}\right) u_{x x}(X, r) \mathrm{d} r\right] \tilde{\varphi}_{\tau} \mathrm{d} s \mathrm{~d} \tau=0 \quad \forall \varphi \in C_{0}^{\infty}\left(Q_{\mathrm{T}}\right) .
$$

Since (4.5) holds for all $\tilde{\varphi} \in C_{0}^{\infty}\left(Q_{\mathrm{T}}\right)$ we have

$$
\begin{gathered}
\frac{\partial}{\partial \tau}\left(\tilde{w} \exp \left[-\int_{0}^{\tau} f^{\prime}\left(u_{x}\right) u_{x x}(X, r) \mathrm{d} r\right]\right)=0 \quad \forall(s, \tau) \in Q_{\mathrm{T}}, \\
\Rightarrow \tilde{w}_{\tau}=f^{\prime}\left(u_{x}\right) \tilde{w} u_{x x}
\end{gathered}
$$

and the result follows since $f^{\prime}\left(u_{x}\right) \tilde{w} \tilde{u}_{x x} \in L^{\infty}\left(Q_{\mathrm{T}}\right)$.

THEOREM 4.1 For $\Omega=(0,1)$, there is at most one solution $\{w, u\}$ of (1.39) and (1.40) with

$$
\begin{gathered}
w, u_{x x} \in L^{\infty}\left(\Omega_{\mathrm{T}}\right), \\
u, u_{x} \in H^{\beta, \beta / 2}\left(\Omega_{\mathrm{T}}\right) \subseteq C\left(\overline{\Omega_{\mathrm{T}}}\right) \quad \text { for some } 0<\beta<1 . \\
w \in L^{\infty}(0, T ; B V(\Omega)) \cap B V\left(\Omega_{\mathrm{T}}\right) .
\end{gathered}
$$

We omit the proof of Theorem 4.1 as it is a straightforward generalization of the proof of Theorem 6.1 in [20].

\section{Steady-state solutions}

We denote the steady-state solutions of $w$ and $u$ by $\tilde{w}$ and $\tilde{u}$, respectively.

Lemma 5.1 Let $\{w, u\}$ solve (1.39) and (1.40) for all $T>0$, with $u(0, t)=u(1, t)=u_{\mathrm{b}}$ and $w(0, t)=w_{\mathrm{b}}$ for all $t \in S_{0}$ and $w(1, t)=w_{\mathrm{b}}$ for all $t \in S_{1}$. Then all points of the $\omega$-limit set

$$
\omega\left(w_{0}\right)=\left\{(\tilde{w}, \tilde{u}): \exists\left\{t_{j}\right\} \text { s.t. } w\left(\cdot, t_{j}\right) \rightarrow \tilde{w} \text { in } L^{1}(\Omega), u\left(\cdot, t_{j}\right) \rightarrow \tilde{u} \text { in } H^{1}(\Omega) \text { as } t_{j} \rightarrow \infty\right\},
$$

(for $1<p<\infty$ ) are steady-state solutions in the sense that 


$$
\begin{array}{cl}
\tilde{w} f\left(\tilde{u}_{x}\right)=0 & \text { in } \Omega, \\
-\lambda^{2} \tilde{u}_{x x}+\tilde{u}=\tilde{w} \geqslant 0 & \text { in } \Omega, \\
\tilde{u}(0)=\tilde{u}(1)=u_{\mathrm{b}}, & \\
\tilde{w}(0)=w_{\mathrm{b}} & \text { if } f\left(\tilde{u}_{x}(0)\right)<0, \\
\tilde{w}(1)=w_{\mathrm{b}} & \text { if } f\left(\tilde{u}_{x}(1)\right)>0 .
\end{array}
$$

We omit the proof of Lemma 5.1 as it is similar to the proof of Lemma 4.1 in [20] and can be found in [11].

LEMMA 5.2 If $w_{0}(x) \equiv 0$ for all $x \in[0,1]$ and $\frac{u_{\mathrm{b}}}{\lambda} \tanh \left(\frac{1}{2 \lambda}\right)<J_{p}$, then no vorticity enters the domain and

$$
w(x, t)=0, \quad \forall(x, t) \in \Omega_{\mathrm{T}} .
$$

Proof. We can explicitly solve $-\lambda^{2} \tilde{u}_{x x}+\tilde{u}=0$ to obtain

$$
\begin{gathered}
u=u_{\mathrm{b}} \cosh \left(\frac{x}{\lambda}\right)-u_{\mathrm{b}} \tanh \left(\frac{1}{2 \lambda}\right) \sinh \left(\frac{x}{\lambda}\right), \\
\Rightarrow u_{x}=\frac{u_{\mathrm{b}}}{\lambda} \sinh \left(\frac{x}{\lambda}\right)-\frac{u_{\mathrm{b}}}{\lambda} \tanh \left(\frac{1}{2 \lambda}\right) \cosh \left(\frac{x}{\lambda}\right) .
\end{gathered}
$$

Since vorticity only enters the sample at inflow boundaries when $v \cdot n \leqslant 0$, i.e. when $\left|u_{x}(0, t)\right|=$ $\left|u_{x}(1, t)\right| \geqslant J_{p}$, from (5.8) if $\left|u_{x}(0, t)\right|=\left|u_{x}(1, t)\right|=\frac{u_{\mathrm{b}}}{\lambda} \tanh \left(\frac{1}{2 \lambda}\right)<J_{p}$, then no vorticity enters the domain and (5.6) holds.

\section{Numerical discretization}

In this section we derive a combined finite volume/element approximation of (1.25)-(1.30) on an interval $[0,1]$ using a uniform grid, with mesh size $h$. We adapt the upwinding method used in [9] where we discretized a simplified version of (1.25)-(1.30) in which $\mathbf{v}=-f\left(u_{x}\right)$ and $w_{\mathrm{b}} \equiv 0$. We set $\Omega=(0,1), h\left(\in R^{+}\right)=\frac{1}{J}, t^{n}=n \Delta t$, and we define $S_{h}, S_{h}^{0}, W_{h}, W_{h}^{0}$ and $a_{h}(\eta, \xi)$, by

$$
\begin{gathered}
S_{h}=\left\{\phi \in C[0,1]:\left.\phi\right|_{[j h,(j+1) h]} \text { is linear } \forall j \in[0, J-1)\right\}, \\
S_{h}^{0}=\left\{\phi \in S_{h}: \phi(0)=\phi(1)=0\right\}, \\
W_{h}=\left\{\eta \in L^{\infty}(0,1): \eta(x)=\eta_{j}, x \in\left(j-\frac{1}{2}\right) h,\left(j+\frac{1}{2}\right) h\right), \forall j \in[1, J-1] \\
\left.\eta(x)=\eta_{0}, x \in\left(0,\left(j-\frac{1}{2}\right) h\right) \text { and } \eta(x)=\eta_{J}, x \in\left(1-\frac{h}{2}, 1\right)\right\}, \\
W_{h}^{0}=\left\{\eta \in W_{h}: \eta_{0}=\eta_{J}=0\right\}, \\
a_{h}(\eta, \xi) \equiv \lambda^{2} \int_{0}^{1} \eta_{x} \xi_{x} \mathrm{~d} x+(\eta, \xi)_{h} \quad \forall \eta, \xi \in H^{1}(0,1),
\end{gathered}
$$


where

$$
(\eta, \xi)_{h}=\frac{h}{2}\left(\eta_{0} \xi_{0}+\eta_{J} \xi_{J}\right)+h \sum_{j=1}^{J-1} \eta_{j} \xi_{j} \quad \forall \eta, \xi \in S_{h} \text { or } W_{h} .
$$

For any $\eta \in S_{h}$, with $\eta_{j}=\eta(j h)$, we have

$$
\eta=\sum_{j=0}^{J} \eta_{j} \xi_{j}(x)
$$

where $\xi_{j} \in S_{h}$ with $\xi_{j}(i h)=\delta_{i j}$ for $0 \leqslant i, j \leqslant J$. For every continuous function $\eta$ on $\bar{\Omega}$ we define the interpolation operator $\pi^{h}: C(\bar{\Omega}) \rightarrow S^{h}$ by $\pi^{h} \eta \in S_{h}$, where $\pi^{h} \eta=\eta$ at every grid point. From [7] we have the following results

$$
\left|\eta-\pi^{h} \eta\right|_{0}+h\left|\eta-\pi^{h} \eta\right|_{1} \leqslant C h^{2} \quad \forall \eta \in H^{2}(\Omega),
$$

where $C$ is a positive constant and

$$
|\eta|_{0}=\left(\int_{\Omega} \eta^{2} \mathrm{~d} x\right)^{\frac{1}{2}}, \quad|\eta|_{1}=\left(\int_{\Omega}\left|\eta_{x}\right|^{2} \mathrm{~d} x\right)^{\frac{1}{2}}
$$

and also that for all $\eta, \xi \in S_{h}^{0}$,

$$
\left|(\eta, \xi)-(\eta, \xi)_{h}\right| \leqslant C h^{2}|\eta|_{1}|\xi|_{1} \leqslant C h|\eta|_{0}|\xi|_{1},
$$

where $|\eta|_{h}=(\eta, \eta)_{h}^{\frac{1}{2}}$. We now define a numerical approximation of (1.25)-(1.30).

$$
\begin{gathered}
w_{j}^{0}=\frac{1}{h} \int_{\left(j-\frac{1}{2}\right) h}^{\left(j+\frac{1}{2}\right) h} w_{0}(x) \mathrm{d} x, \quad \forall j \in[1, J-1], \\
w_{0}^{0}=\frac{h}{2} \int_{0}^{\frac{1}{2 h}} w_{0}(x) \mathrm{d} x, \quad w_{J}^{0}=\frac{h}{2} \int_{1-\frac{1}{2 h}}^{1} w_{0}(x) \mathrm{d} x, \\
w_{h}^{0}(x)=\sum_{j=0}^{J} w_{j}^{0} \xi_{j}(x) \in S_{h}, \\
\hat{w}_{h}^{0}(x) \in W_{h} . \\
a_{h}\left(u_{h}^{n}, \xi\right)=\left(\hat{w}_{h}^{n}, \xi\right)_{h} \quad \forall \xi \in S_{h}^{0}, \\
u_{0}^{n}=u_{J}^{n}=u_{\mathrm{b}},
\end{gathered}
$$

where $\hat{w}_{h}^{n}-w_{\mathrm{b}}=\sum_{j=0}^{J} w_{j}^{n} \xi_{j}(x)-w_{\mathrm{b}} \in S_{h}^{0}$ and $u_{h}^{n}-u_{\mathrm{b}}=\sum_{j=0}^{J} u_{j}^{n} \xi_{j}(x)-u_{\mathrm{b}} \in S_{h}^{0}$. Note that (6.7) can be written explicitly as;

$$
\lambda^{2}\left(q_{j}^{n}-q_{j-1}^{n}\right)=h\left(u_{j}^{n}-w_{j}^{n}\right) \quad \forall j \in[1, J-1],
$$


where $q_{j}^{n}=\frac{1}{h}\left(u_{j+1}^{n}-u_{j}^{n}\right)$. Adapting the semi-implicit upwinding scheme in [9] we arrive at the following discretization of (1.25)

$$
w_{j}^{n+1}=w_{j}^{n}+\frac{\Delta t}{h}\left(w_{j+1}^{n+1}\left[f_{j}^{n}\right]_{+}+w_{j}^{n+1}\left[f_{j}^{n}\right]_{-}-w_{j}^{n+1}\left[f_{j-1}^{n}\right]_{+}-w_{j-1}^{n+1}\left[f_{j-1}^{n}\right]_{-}\right),
$$

for all $j \in[1, J-1]$, where $f_{j}^{n}:=f\left(q_{j}^{n}\right)$. From (1.32) we have

$$
\left|f_{j}^{n}\right| \leqslant \mu\left|q_{j}^{n}\right|, \quad\left|f_{j}^{n}-f_{j-1}^{n}\right| \leqslant \mu\left|q_{j}^{n}-q_{j-1}^{n}\right|, \quad 0 \leqslant f[r, s], \quad|f[r, s, t]| \leqslant C,
$$

where $f[r, s]=(f(s)-f(r)) /(s-r)$ and $f[r, s, t]=(f[s, t]-f[r, s]) /(t-r)$ are, respectively, the first and second divided differences of $f$. Finally we set

$$
w_{0}^{n}=w_{J}^{n}=w_{\mathrm{b}} \quad \forall n \leqslant 0 .
$$

From (6.9) and (6.11) we see that if $\left(f_{j-1}^{n}-f_{j}^{n}\right) \leqslant 0$, we have

$$
0 \leqslant\left(f_{j}^{n}-f_{j-1}^{n}\right) \leqslant \mu\left(q_{j}^{n}-q_{j-1}^{n}\right) \leqslant \mu h \lambda^{-2}\left(u_{j}^{n}-w_{j}^{n}\right) \leqslant \mu h \lambda^{-2} u_{j}^{n} .
$$

Lemma 6.1 Set $\Lambda=\max \left(\left\|w_{h}^{0}\right\|_{\infty}, u_{\mathrm{b}}, w_{\mathrm{b}}\right)$. Then for all $h>0$ and $0<\Delta t<\frac{\lambda^{2}}{\mu \Lambda}$, there exists a unique sequence $\left\{\hat{w}_{h}^{n}, u_{h}^{n}\right\}$, which for $u_{h}^{n}$ solves (6.7) for all $n \geqslant 0$ and $\hat{w}_{h}^{n}$ solves (6.10) for $n \geqslant 1$. Also we have

$$
\begin{gathered}
0 \leqslant w_{j}^{n} \leqslant \max _{j \in[0, J]} w_{j}^{n} \leqslant \Lambda, \\
0 \leqslant u_{j}^{n} \leqslant \max _{j \in[0, J]} u_{j}^{n} \leqslant \Lambda, \\
\left\|u_{h}^{n}\right\|_{a}^{2} \leqslant C,
\end{gathered}
$$

for $n \geqslant 0$, where $\|u\|_{a}^{2}=a_{h}(u, u)$ and $C$ is a constant depending on the initial data $w_{h}^{0}$ and the boundary data $u_{\mathrm{b}}$ and $w_{\mathrm{b}}$.

Proof. We omit proving that if (6.14) holds for any $n$ then there exists a unique $u_{h}^{n}$ satisfying (6.15) for that $n$, since it follows directly from the proof of Lemma 2.2 in [9]. We prove (6.14) by induction. Firstly we rewrite (6.10) as

$$
\left(1+\frac{\Delta t}{h}\left(\left[f_{j-1}^{n}\right]_{+}-\left[f_{j}^{n}\right]_{-}\right)\right) w_{j}^{n+1}+\frac{\Delta t}{h}\left(w_{j+1}^{n+1}\left[f^{n}\right]_{+}-w_{j-1}^{n-1}\left[f_{j-1}^{n}\right]_{-}\right)=w_{j}^{n}
$$

which is a system of equations of the form

$$
L\left(u_{h}^{n}\right) \underline{w}_{h}^{n+1}=\underline{w}_{h}^{n},
$$

where $\underline{w}_{h}^{n}=\left(w_{j}^{n}, \forall j \in[1, J-1]\right)^{T}$ and $L\left(u_{h}^{n}\right)$ is a matrix with diagonal elements $L_{j j} \geqslant 1$, and off-diagonal elements $L_{j k} \leqslant 0$, for all $j \neq k$. Since $L_{j j}-\sum_{k \neq j}\left|L_{j k}\right|=1+\frac{\Delta t}{h}\left(f_{j-1}^{n}-f_{j}^{n}\right)$, if $\left(f_{j-1}^{n}-f_{j}^{n}\right)>0$ we have $L_{j j}-\sum_{k \neq j}\left|L_{j k}\right|>1$. However, if $\left(f_{j-1}^{n}-f_{j}^{n}\right) \leqslant 0$, from (6.13) it follows that since $\Delta t<\frac{\lambda^{2}}{\mu \Lambda}$, we have $\Delta t \mu\left\|u_{h}^{n}\right\|_{\infty}<\lambda^{2}$ and thus the matrix $L$ is strictly diagonally dominant and therefore invertible, giving a unique solution $\bar{w}_{h}^{n+1}$. We now prove (6.14) by induction. 
Trivially the result holds if $n=0$, now we assume the result holds for $n$ and then prove it holds for $n+1$. Firstly we note that $L$ is an M-matrix [21], and hence $L\left(u_{h}^{n}\right)^{-1}$ has only non-negative elements, thus from (6.18) it follows that

$$
w_{h}^{n} \geqslant 0 \Rightarrow w_{h}^{n+1} \geqslant 0 .
$$

We now prove the second part of (6.14) by contradiction and suppose that there exists an integer $j_{0} \in[1, J-1]$ such that

$$
w_{j_{0}}^{n+1}=\max _{j \in[1, J-1]} w_{j}^{n+1}>\max \left(w_{j}^{0}, u_{\mathrm{b}}, \alpha\right) .
$$

Writing (6.17) using the index $j_{0}$ gives

$$
w_{j_{0}}^{n+1}\left[1-\frac{\Delta t}{h}\left(f_{j_{0}}^{n}-f_{j_{0}-1}^{n}\right)\right] \leqslant w_{j_{0}}^{n},
$$

and hence if $\left(f_{j_{0}}^{n}-f_{j_{0}-1}^{n}\right)<0$, then (6.14) holds. However, if $\left(f_{j_{0}}^{n}-f_{j_{0}-1}^{n}\right) \geqslant 0$, using (6.11) and (6.20), we have that

$$
w_{j_{0}}^{n+1}\left[1-\frac{\mu \Delta t}{h}\left(q_{j_{0}}^{n}-q_{j_{0}-1}^{n}\right)\right] \leqslant w_{j_{0}}^{n} \Rightarrow w_{j_{0}}^{n+1}\left(1+\frac{\mu \Delta t}{\lambda^{2}}\left(w_{j_{0}}^{n}-u_{j_{0}}^{n}\right)\right) \leqslant w_{j_{0}}^{n} .
$$

From (6.21) we see that if $w_{j_{0}}^{n} \geqslant u_{j_{0}}^{n}$, then we obtain a contradiction to (6.19). Thus it remains to consider the case where

$$
w_{j_{0}}^{n}<u_{j_{0}}^{n} .
$$

Since $\mu \Lambda \Delta t \lambda^{-2}<1$, if $w_{j_{0}}^{n}<u_{j_{0}}^{n}$, then (6.19) and (6.21) imply

$$
w_{j_{0}}^{n}>\Lambda \Delta t\left(w_{j_{0}}^{n}-u_{j_{0}}^{n}\right)>\Lambda+w_{j_{0}}^{n}-u_{j_{0}}^{n},
$$

which contradicts $u_{j_{0}}^{n} \leqslant \Lambda$. Thus (6.14) holds for $n=n+1$ and by induction (6.14) holds for all $n \geqslant 0$.

To prove (6.16) we set $\chi=u_{h}^{n}-u_{\mathrm{b}}$ in (6.10) and use (6.14) and (6.15) to obtain

$$
\begin{gathered}
a_{h}\left(u_{h}^{n}, u_{h}^{n}\right)=a_{h}\left(u_{h}^{n}, u_{\mathrm{b}}\right)+\left(w_{h}^{n}, u_{h}^{n}-u_{\mathrm{b}}\right)_{h} \leqslant C \\
\Rightarrow\left\|u_{h}^{n}\right\|_{a}^{2}=\lambda^{2} \int_{\Omega}\left|\left(u_{h}^{n}\right)_{x}\right|^{2} \mathrm{~d} x+\left(u_{h}^{n}, u_{h}^{n}\right)_{h}=a_{h}\left(u_{h}^{n}, u_{h}^{n}\right) \leqslant C .
\end{gathered}
$$

This completes the proof.

REMARK 6.1 For Lemma 6.1 to hold we require

$$
\Delta t<\frac{\lambda^{2}}{\mu \max \left(\left\|w_{h}^{0}\right\|_{L^{\infty}(\Omega)}, u_{\mathrm{b}}\right)}=\frac{\lambda^{2}}{\mu \Lambda},
$$

and from here onwards we assume $\Delta t$ satisfies (6.23). 
LEMmA 6.2 There exist constants $C$ independent of $h$ and $\Delta t$, such that for all $n \geqslant 0$

$$
\begin{array}{r}
\left|f_{j}^{n}\right| \leqslant \frac{C}{\lambda^{2}}, \\
\frac{\left|q_{j+1}^{n}-q_{j}^{n}\right|}{h} \leqslant \frac{C}{\lambda^{2}} .
\end{array}
$$

Proof. For the proof of this lemma see the proof of Lemma 5.6 [9].

LEMma 6.3 Let $0<\varepsilon<2 \lambda^{2} / \mu$. Then for all $\Delta t<\frac{\varepsilon}{2 \Lambda}$, and $n \geqslant 0$, we have

$$
E_{h}\left(u_{h}^{n+1}-u_{\mathrm{b}}\right)+\left(1 / \mu-\frac{\varepsilon}{2 \lambda^{2}}\right) \Delta t \sum_{j=0}^{J-1} h\left(w_{j+1}^{n+1}\left[f_{j}^{n}\right]_{+}^{2}+w_{j}^{n+1}\left[f_{j}^{n}\right]_{-}^{2}\right) \leqslant E_{h}\left(u_{h}^{n}-u_{\mathrm{b}}\right),
$$

where

$$
E_{h}(v)=1 / 2\|v\|_{a}^{2}=1 / 2 a_{h}(v, v) .
$$

Proof. Setting $\chi \equiv u_{h}^{n+1}-u_{\mathrm{b}}$ in (6.7), using (6.1) and noting that $u_{0}^{n}=u_{J}^{n}=u_{\mathrm{b}}$ for all $n \geqslant 0$, gives

$$
\begin{aligned}
a_{h}\left(u_{h}^{n+1}-u_{h}^{n}, u_{h}^{n+1}-u_{\mathrm{b}}\right)= & \left(w_{h}^{n+1}-w_{h}^{n}, u_{h}^{n+1}-u_{\mathrm{b}}\right)_{h} \\
= & \Delta t \sum_{j=0}^{J-1}\left(w_{j+1}^{n+1}\left[f_{j}^{n}\right]_{+}+w_{j}^{n+1}\left[f_{j}^{n}\right]_{-}\right)\left(u_{j}^{n+1}-u_{\mathrm{b}}\right) \\
& -\sum_{j=1}^{J}\left(w_{j}^{n+1}\left[f_{j-1}^{n}\right]_{+}+w_{j-1}^{n+1}\left[f_{j-1}^{n}\right]_{-}\right)\left(u_{j}^{n+1}-u_{\mathrm{b}}\right) \\
= & \Delta t \sum_{j=0}^{J-1}\left(w_{j+1}^{n+1}\left[f_{j}^{n}\right]_{+}+w_{j}^{n+1}\left[f_{j}^{n}\right]_{-}\right)\left(u_{j}^{n+1}-u_{j+1}^{n+1}\right) \\
= & \Delta t \sum_{j=0}^{J-1}\left(w_{j+1}^{n+1}\left[f_{j}^{n}\right]_{+}+w_{j}^{n+1}\left[f_{j}^{n}\right]_{-}\right)\left(u_{j}^{n}-u_{j+1}^{n}\right) \\
& +\Delta t \sum_{j=0}^{J-1}\left(w_{j+1}^{n+1}\left[f_{j}^{n}\right]_{+}+w_{j}^{n+1}\left[f_{j}^{n}\right]_{-}\right)\left(u_{j}^{n+1}-u_{j+1}^{n+1}\right) \\
& -\Delta t \sum_{j=0}^{J-1}\left(w_{j+1}^{n+1}\left[f_{j}^{n}\right]_{+}+w_{j}^{n+1}\left[f_{j}^{n}\right]_{-}\right)\left(u_{j}^{n}-u_{j+1}^{n}\right) .
\end{aligned}
$$

Setting $\|\bar{u}\|_{a}^{2}=a_{h}\left(u-u_{\mathrm{b}}, u-u_{\mathrm{b}}\right)$ and rearranging gives

$$
\begin{aligned}
\left\|\bar{u}_{h}^{n+1}\right\|_{a}^{2}+\| \bar{u}_{h}^{n+1} & -\bar{u}_{h}^{n} \|_{a}^{2} \\
& \leqslant\left\|\bar{u}_{h}^{n}\right\|_{a}^{2}-2 h \Delta t \sum_{j=0}^{J-1}\left(w_{j+1}^{n+1}\left[f_{j}^{n}\right]_{+}+w_{j}^{n+1}\left[f_{j}^{n}\right]_{-}\right) q_{j}^{n}
\end{aligned}
$$




$$
\begin{aligned}
& +\frac{\lambda^{2} \Delta t}{h \varepsilon} \sum_{j=0}^{J-1}\left(w_{j+1}^{n+1}+w_{j}^{n+1}\right)\left(\left(u_{j}^{n+1}-u_{j+1}^{n+1}\right)-\left(u_{j}^{n}-u_{j+1}^{n}\right)\right)^{2} \\
& +\frac{h \varepsilon \Delta t}{\lambda^{2}} \sum_{j=0}^{J-1}\left(w_{j+1}^{n+1}\left[f_{j}^{n}\right]_{+}^{2}+w_{j}^{n+1}\left[f_{j}^{n}\right]_{-}^{2}\right) .
\end{aligned}
$$

Noting that $f_{j}>0 \Rightarrow q_{j}>0$ and $f_{j}<0 \Rightarrow q_{j}<0$, from (6.28) we have

$$
\begin{aligned}
\left\|\bar{u}_{h}^{n+1}\right\|_{a}^{2}+\| \bar{u}_{h}^{n+1}- & \bar{u}_{h}^{n} \|_{a}^{2} \\
\leqslant & \left\|\bar{u}_{h}^{n}\right\|_{a}^{2}-2 h \Delta t \sum_{j=0}^{J-1}\left(w_{j+1}^{n+1}\left[f_{j}^{n}\right]_{+}\left[q_{j}^{n}\right]_{+}+w_{j}^{n+1}\left[f_{j}^{n}\right]_{-}\left[q_{j}^{n}\right]_{-}\right) \\
& +\frac{\lambda^{2} \Delta t}{h \varepsilon} \sum_{j=0}^{J-1}\left(w_{j+1}^{n+1}+w_{j}^{n+1}\right)\left(\left(u_{j}^{n+1}-u_{j+1}^{n+1}\right)-\left(u_{j}^{n}-u_{j+1}^{n}\right)\right)^{2} \\
& +\frac{h \varepsilon \Delta t}{\lambda^{2}} \sum_{j=0}^{J-1}\left(w_{j+1}^{n+1}\left[f_{j}^{n}\right]_{+}^{2}+w_{j}^{n+1}\left[f_{j}^{n}\right]_{-}^{2}\right) \\
\leqslant & \left\|\bar{u}_{h}^{n}\right\|_{a}^{2}-\frac{2 h \Delta t}{\mu} \sum_{j=0}^{J-1}\left(w_{j+1}^{n+1}\left[f_{j}^{n}\right]_{+}^{2}+w_{j}^{n+1}\left[f_{j}^{n}\right]_{-}^{2}\right) \\
& +\frac{\lambda^{2} \Delta t}{h \varepsilon} \sum_{j=0}^{J-1}\left(w_{j+1}^{n+1}+w_{j}^{n+1}\right)\left(\left(u_{j}^{n+1}-u_{j+1}^{n+1}\right)-\left(u_{j}^{n}-u_{j+1}^{n}\right)\right)^{2} \\
& +\frac{h \varepsilon \Delta t}{\lambda^{2}} \sum_{j=0}^{J-1}\left(w_{j+1}^{n+1}\left[f_{j}^{n}\right]_{+}^{2}+w_{j}^{n+1}\left[f_{j}^{n}\right]_{-}^{2}\right) .
\end{aligned}
$$

Since

$$
\left\|\bar{u}_{h}^{n}\right\|_{a}^{2}=\frac{\lambda^{2}}{h} \sum_{j=0}^{J-1}\left(\left(u_{j}^{n+1}-u_{j+1}^{n+1}\right)-\left(u_{j}^{n}-u_{j+1}^{n}\right)\right)^{2}+\sum_{j=0}^{J} h\left(u_{j}^{n}-u_{\mathrm{b}}\right)^{2}
$$

it follows that

$$
\begin{aligned}
\left\|\bar{u}_{h}^{n+1}\right\|_{a}^{2}+\left(\frac{2}{\mu}-\frac{\varepsilon}{\lambda^{2}}\right) h \Delta t \sum_{j=0}^{J-1}\left(w_{j+1}^{n+1}\left[f_{j}^{n}\right]_{+}^{2}\right. & \left.+w_{j}^{n+1}\left[f_{j}^{n}\right]_{-}^{2}\right) \\
& \leqslant\left\|\bar{u}_{h}^{n}\right\|_{a}^{2}+\left(\frac{2\left\|w_{h}^{n+1}\right\|_{\infty} \Delta t}{\varepsilon}-1\right)\left\|\bar{u}_{h}^{n+1}-\bar{u}_{h}^{n}\right\|_{a}^{2} .
\end{aligned}
$$

Taking $\varepsilon$ and $\Delta t$ such that $\left(\frac{2}{\mu}-\frac{\varepsilon}{\lambda^{2}}\right)>0$ and $\left(\frac{2 \Delta t \Lambda}{\varepsilon}-1\right)<0$ gives the required result.

We omit the proof of the following lemma as it is essentially the same as the proof of Lemma 5.5 in [9] with $q_{j}^{n}$ replaced by $f_{j}^{n}$ and can be found in [11].

LEMma 6.4 There exists a constant $C$ independent of $h$ and $\Delta t$, such that

$$
\sum_{n=0}^{N} \frac{1}{\Delta t}\left\|u_{h}^{n+1}-u_{h}^{n}\right\|_{a}^{2} \leqslant C
$$


Lemma 6.5 Set $\Lambda^{*}>\Lambda$ to be a constant. Then for all $\Delta t \leqslant \min \left(\frac{\lambda^{2}}{\mu \Lambda}, \frac{\Lambda^{*}-\Lambda}{\Lambda^{*} \Lambda}\right)$ and all $n \geqslant 0$ we have

$$
\left|\hat{w}_{h}^{n}\right|_{T V[0, L]}=\sum_{j=0}^{J-1}\left|w_{j+1}^{n}-w_{j}^{n}\right| \leqslant C_{1}\left(\left|\hat{w}_{h}^{0}\right|_{T V[0, L]}+C\left(\lambda^{-4}+\mu \lambda^{-2}\right)(n+1) \Delta t\right),
$$

where

$$
C_{1}=e^{(n+1) \Lambda^{*} \Delta t}
$$

Proof. From (6.10) we see that for $j=1, \ldots, J-1$

$$
\begin{aligned}
w_{j}^{n+1}= & w_{j}^{n}+\frac{\Delta t}{h}\left(w_{j+1}^{n+1}\left[f_{j}^{n}\right]_{+}+w_{j}^{n+1}\left[f_{j}^{n}\right]_{-}-w_{j}^{n+1}\left[f_{j-1}^{n}\right]_{+}-w_{j-1}^{n+1}\left[f_{j-1}^{n}\right]_{-}\right) \\
= & w_{j}^{n}+\frac{\Delta t}{h}\left(\left(w_{j+1}^{n+1}-w_{j}^{n+1}\right)\left[f_{j}^{n}\right]_{+}+\left(w_{j}^{n+1}-w_{j-1}^{n+1}\right)\left[f_{j-1}^{n}\right]_{-}\right) \\
& +\frac{\Delta t}{h} w_{j}^{n+1}\left(f_{j}^{n}-f_{j-1}^{n}\right) .
\end{aligned}
$$

Setting $\xi_{j}^{n}=w_{j+1}^{n}-w_{j}^{n}$ gives the following for $j=1, \ldots, J-2$

$$
\begin{aligned}
\xi_{j}^{n+1}=\xi_{j}^{n}+ & \frac{\Delta t}{h}\left(\xi_{j+1}^{n+1}\left[f_{j+1}\right]_{+}+w_{j+1}^{n+1}\left(f_{j+1}^{n}-f_{j}^{n}\right)+\xi_{j}^{n+1}\left[f_{j}^{n}\right]_{-}-\xi_{j}^{n+1}\left[f_{j}^{n}\right]_{+}\right) \\
& -\frac{\Delta t}{h} w_{j}^{n+1}\left(f_{j}^{n}-f_{j-1}^{n}\right)-\frac{\Delta t}{h} \xi_{j-1}^{n+1}\left[f_{j-1}^{n}\right]_{-} \\
\Rightarrow\left(1+\frac{\Delta t}{h}\left[f_{j}^{n}\right]_{+}-\frac{\Delta t}{h}\left[f_{j}^{n}\right]_{-}\right) \xi_{j}^{n+1}= & \xi_{j}^{n}+\frac{\Delta t}{h} \xi_{j+1}^{n+1}\left[f_{j+1}^{n}\right]_{+}-\frac{\Delta t}{h} \xi_{j-1}^{n+1}\left[f_{j-1}^{n}\right]_{-} \\
& +\frac{\Delta t}{h}\left(w_{j+1}^{n}-w_{j}^{n}\right)\left(f_{j+1}^{n}-f_{j}^{n}\right) \\
& +\frac{\Delta t}{h} w_{j}^{n+1}\left(f_{j+1}^{n}-2 f_{j}^{n}+f_{j-1}^{n}\right) .
\end{aligned}
$$

Using the divided difference notation of (6.11) we see that

$$
\begin{aligned}
f_{j+1}^{n}-2 f_{j}^{n}+f_{j-1}^{n}= & f\left[q_{j+1}^{n}, q_{j}^{n}\right]\left(q_{j+1}^{n}-q_{j}^{n}\right)-f\left[q_{j}^{n}, q_{j-1}^{n}\right]\left(q_{j}^{n}-q_{j-1}^{n}\right) \\
= & \left(q_{j+1}^{n}-q_{j}^{n}\right)\left(f\left[q_{j+1}^{n}, q_{j}^{n}\right]-f\left[q_{j}^{n}, q_{j-1}^{n}\right]\right) \\
& +f\left[q_{j}^{n}, q_{j-1}^{n}\right]\left(q_{j+1}^{n}-2 q_{j}^{n}+q_{j-1}^{n}\right) \\
= & \left(q_{j+1}^{n}-q_{j}^{n}\right) f\left[q_{j+1}^{n}, q_{j}^{n}, q_{j-1}^{n}\right]\left(q_{j+1}^{n}-q_{j-1}^{n}\right) \\
& +f\left[q_{j}^{n}, q_{j-1}^{n}\right]\left(q_{j+1}^{n}-2 q_{j}^{n}+q_{j-1}^{n}\right) \\
= & h^{2} \lambda^{-4}\left(u_{j+1}^{n}-w_{j+1}^{n}\right)\left(u_{j+1}^{n}-w_{j+1}^{n}+\left(u_{j}^{n}-w_{j}^{n}\right)\right) f\left[q_{j+1}^{n}, q_{j}^{n}, q_{j-1}^{n}\right] \\
& +h \lambda^{-2}\left(\left(u_{j+1}^{n}-u_{j}^{n}\right)-\left(w_{j+1}^{n}-w_{j}^{n}\right)\right) f\left[q_{j}^{n}, q_{j-1}^{n}\right] \\
= & h^{2} \lambda^{-4}\left(u_{j+1}^{n}-w_{j+1}^{n}\right)^{2} f\left[q_{j+1}^{n}, q_{j}^{n}, q_{j-1}^{n}\right] \\
& +h^{2} \lambda^{-4}\left(u_{j+1}^{n}-w_{j+1}^{n}\right)\left(u_{j}^{n}-w_{j}^{n}\right) f\left[q_{j+1}^{n}, q_{j}^{n}, q_{j-1}^{n}\right] \\
& +h^{2} \lambda^{-2} q_{j}^{n} f\left[q_{j}^{n}, q_{j-1}^{n}\right]-h \lambda^{-2} \xi_{j}^{n} f\left[q_{j}^{n}, q_{j-1}^{n}\right] .
\end{aligned}
$$


Using (6.34) in (6.33) gives the following for $j=1, \ldots, J-2$,

$$
\begin{aligned}
\left(1+\frac{\Delta t}{h}\left[f_{j}^{n}\right]_{+}-\right. & \left.\frac{\Delta t}{h}\left[f_{j}^{n}\right]_{-}-\frac{\Delta t}{h}\left(f_{j+1}^{n}-f_{j}^{n}\right)\right) \xi_{j}^{n+1} \\
= & \left(1-\Delta t \lambda^{-2} w_{j}^{n+1} f\left[q_{j}^{n}, q_{j-1}^{n}\right]\right) \xi_{j}^{n}+\frac{\Delta t}{h} \xi_{j+1}^{n+1}\left[f_{j+1}^{n}\right]_{+} \\
& -\frac{\Delta t}{h} \xi_{j-1}^{n+1}\left[f_{j-1}^{n}\right]_{-}+\Delta t \lambda^{-2} h w_{j}^{n+1} q_{j}^{n} f\left[q_{j}^{n}, q_{j-1}^{n}\right] \\
& +\Delta t \lambda^{-4} h w_{j}^{n+1}\left(u_{j+1}^{n}-w_{j+1}^{n}\right)\left(u_{j}^{n}-w_{j}^{n}\right) f\left[q_{j+1}^{n}, q_{j}^{n}, q_{j-1}^{n}\right] \\
& +\Delta t h \lambda^{-4} w_{j}^{n+1}\left(u_{j+1}^{n}-w_{j+1}^{n}\right)^{2} f\left[q_{j+1}^{n}, q_{j}^{n}, q_{j-1}^{n}\right] .
\end{aligned}
$$

From Lemma 6.1 and (6.11) we see that all the coefficients of $\xi$ in the above equation are positive and hence for $j=1, \ldots, J-2$ we have

$$
\begin{aligned}
(1+ & \left.\frac{\Delta t}{h}\left[f_{j}^{n}\right]_{+}-\frac{\Delta t}{h}\left[f_{j}^{n}\right]_{-}-\frac{\Delta t}{h}\left(f_{j+1}^{n}-f_{j}^{n}\right)\right)\left|\xi_{j}^{n+1}\right| \\
\leqslant & \left|\xi_{j}^{n}\right|+\frac{\Delta t}{h}\left|\xi_{j+1}^{n+1}\right|\left[f_{j+1}^{n}\right]_{+}-\frac{\Delta t}{h}\left|\xi_{j-1}^{n+1}\right|\left[f_{j-1}^{n}\right]_{-}+\Delta t h \lambda^{-2} w_{j}^{n+1}\left|q_{j}^{n}\right| f\left[q_{j}^{n}, q_{j-1}^{n}\right] \\
& +\Delta t h \lambda^{-4}\left(\left(u_{j+1}^{n}-w_{j+1}^{n}\right)^{2}+\left|\left(u_{j+1}^{n}-w_{j+1}^{n}\right)\left(u_{j}^{n}-w_{j}^{n}\right)\right|\right)\left|f\left[q_{j+1}^{n}, q_{j}^{n}, q_{j-1}^{n}\right]\right| .
\end{aligned}
$$

Summing the above inequality from $j=1$ to $J-2$, using Lemma 6.1 and (6.11), gives

$$
\begin{aligned}
(1-\Lambda \Delta t) \sum_{j=1}^{J-2}\left|\xi_{j}^{n+1}\right| \leqslant & \sum_{j=1}^{J-2}\left|\xi_{j}^{n}\right|+\frac{\Delta t}{h} \sum_{j=1}^{J-2}\left(\left|\xi_{j+1}^{n+1}\right|\left[f_{j+1}^{n}\right]_{+}-\left|\xi_{j-1}^{n+1}\right|\left[f_{j-1}^{n}\right]_{-}\right) \\
& -\frac{\Delta t}{h} \sum_{j=1}^{J-2}\left|\xi_{j}^{n+1}\right|\left(\left[f_{j}^{n}\right]_{+}-\left[f_{j}^{n}\right]_{-}\right)+C\left(\lambda^{-4}+\mu \lambda^{-2}\right) \Delta t \sum_{j=1}^{J-2} h \\
= & \sum_{j=1}^{J-2}\left|\xi_{j}^{n}\right|+C\left(\lambda^{-4}+\mu \lambda^{-2}\right) \Delta t \sum_{j=1}^{J-2} h \\
& +\frac{\Delta t}{h}\left(\left|\xi_{J-1}^{n+1}\right|\left[f_{J-1}^{n}\right]_{+}-\left|\xi_{1}^{n+1}\right|\left[f_{1}^{n}\right]_{+}\right) \\
& +\frac{\Delta t}{h}\left(\left|\xi_{J-2}^{n+1}\right|\left[f_{J-2}^{n}\right]_{-}-\left|\xi_{0}^{n+1}\right|\left[f_{0}^{n}\right]_{-}\right) .
\end{aligned}
$$

Using (6.12) and (6.32) we have

$$
\begin{aligned}
& \xi_{0}^{n+1}=\xi_{0}^{n}+\frac{\Delta t}{h}\left(\xi_{1}^{n+1}\left[f_{1}^{n}\right]_{+}+w_{1}^{n+1}\left(f_{1}^{n}-f_{0}^{n}\right)+\xi_{0}^{n+1}\left[f_{0}^{n}\right]_{-}\right), \\
& \xi_{J-1}^{n+1}=\xi_{J-1}^{n}-\frac{\Delta t}{h}\left(\xi_{J-1}^{n+1}\left[f_{J-1}^{n}\right]_{+}+w_{J-1}^{n+1}\left(f_{J-1}^{n}-f_{J-2}^{n}\right)+\xi_{J-2}^{n+1}\left[f_{J-2}^{n}\right]_{-}\right) .
\end{aligned}
$$

and from (6.11) it follows that

$$
\begin{gathered}
(1-\Lambda \Delta t)\left|\xi_{0}^{n+1}\right| \leqslant \\
+\xi_{0}^{n}\left|+\frac{\Delta t}{h}\right| \xi_{1}^{n+1}\left|\left[f_{1}^{n}\right]_{+}+\frac{\Delta t}{h}\right| \xi_{0}^{n+1} \mid\left[f_{0}^{n}\right]_{-} \\
+C \mu \lambda^{-2} \Delta t\left|w_{1}^{n+1}\left(w_{1}^{n}-u_{1}^{n}\right)\right|
\end{gathered}
$$




$$
\begin{gathered}
(1-\Lambda \Delta t)\left|\xi_{J-1}^{n+1}\right| \leqslant \\
+\xi_{J-1}^{n}\left|-\frac{\Delta t}{h}\right| \xi_{J-2}^{n+1}\left|\left[f_{J-2}^{n}\right]_{-}-\frac{\Delta t}{h}\right| \xi_{J-1}^{n+1} \mid\left[f_{J-1}^{n}\right]_{+} \\
+C \mu \lambda^{-2} \Delta t\left|w_{J-1}^{n+1}\left(w_{J-1}^{n}-u_{J-1}^{n}\right)\right| .
\end{gathered}
$$

The above definitions of $\left|\xi_{0}^{n+1}\right|$ and $\left|\xi_{J-1}^{n+1}\right|$ coupled with (6.35) and the fact that $\Delta t \leqslant \frac{C^{*}-\Lambda}{C^{*} \Lambda}$, we obtain

$$
\begin{aligned}
\sum_{j=0}^{J-1}\left|\xi_{j}^{n+1}\right| & \leqslant \frac{1}{1-\Lambda \Delta t}\left(\sum_{j=0}^{J-1}\left|\xi_{j}^{n}\right|+C\left(\lambda^{-4}+\mu \lambda^{-2}\right) \Delta t\right) \\
& \leqslant\left(1+\Lambda^{*} \Delta t\right)\left(\sum_{j=0}^{J-1}\left|\xi_{j}^{n}\right|+C\left(\lambda^{-4}+\mu \lambda^{-2}\right) \Delta t\right) \\
\Rightarrow \sum_{j=0}^{J-1}\left|\xi_{j}^{n+1}\right| & \leqslant\left(1+\Lambda^{*} \Delta t\right)^{n+1} \sum_{j=0}^{J-1}\left|\xi_{j}^{0}\right|+C \sum_{j=0}^{n}\left(1+\Lambda^{*} \Delta t\right)^{j} \Delta t \\
& \leqslant\left(1+\Lambda^{*} \Delta t\right)^{n+1} \sum_{j=0}^{J-1}\left|\xi_{j}^{0}\right|+(n+1)\left(1+\Lambda^{*} \Delta t\right)^{n+1} C\left(\lambda^{-4}+\mu \lambda^{-2}\right) \Delta t, \\
\Rightarrow \sum_{j=0}^{J-1}\left|\xi_{j}^{n+1}\right| & \leqslant e^{(n+1) \Lambda^{*} \Delta t}\left(\sum_{j=0}^{J-1}\left|\xi_{j}^{0}\right|+C\left(\lambda^{-4}+\mu \lambda^{-2}\right)(n+1) \Delta t\right),
\end{aligned}
$$

and the result follows.

Lemma 6.6 There exists a constant $C$ independent of $h$ and $\Delta t$, such that

$$
\begin{gathered}
\left.\| \hat{w}_{h}^{n+1}-\left.\hat{w}_{h}^{n}\right|_{L^{1}(0, L)}=\sum_{j=1}^{J-1} h\left|w_{j}^{n+1}-w_{j}^{n}\right| \leqslant C\left(\lambda^{-4}+\mu \lambda^{-2}\right)\right) \Delta t \quad \forall n \geqslant 0, \\
\left|q_{j}^{n+1}-q_{j}^{n}\right| \leqslant C \Delta t \quad \forall j \in[0, J-1], n \geqslant 0 .
\end{gathered}
$$

Proof. We omit the proof of (6.36) as it is a straightforward generalization of the proof of Lemma 5.8 in [9]. To prove (6.37) we set $\xi=u_{h}^{n+1}-u_{h}^{n}$ in (6.7) to obtain

$$
a_{h}\left(u_{h}^{n+1}-u_{h}^{n}, u_{h}^{n+1}-u_{h}^{n}\right)=\left(\hat{w}_{h}^{n+1}-\hat{w}_{h}^{n}, u_{h}^{n+1}-u_{h}^{n}\right)_{h}
$$

noting that in one dimension and $\|\cdot\|_{\infty} \leqslant C\|\cdot\|_{H^{1}}$, we have

$$
\begin{aligned}
\left\|u_{h}^{n+1}-u_{h}^{n}\right\|_{a}^{2} & \leqslant\left\|\hat{w}_{h}^{n+1}-\hat{w}_{h}^{n}\right\|_{L^{1}(\Omega)}\left\|u_{h}^{n+1}-u_{h}^{n}\right\|_{L^{\infty}(\Omega)} \\
\Rightarrow\left\|u_{h}^{n+1}-u_{h}^{n}\right\|_{L^{\infty}(\Omega)} & \leqslant C\left\|\hat{w}_{h}^{n+1}-\hat{w}_{h}^{n}\right\|_{L^{1}(\Omega)} .
\end{aligned}
$$

Replacing $q_{j}^{n}$ with $q_{j}^{n+1}-q_{j}^{n}$ in the proof of Lemma 5.6 in [9] we obtain

$$
\begin{aligned}
J\left(q_{j}^{n+1}-q_{j}^{n}\right)= & -\sum_{k=j+1}^{J-1} \sum_{i=j+1}^{k} h\left(u_{i}^{n+1}-u_{i}^{n}-w_{i}^{n+1}+w_{i}^{n}\right) \\
& +\sum_{k=0}^{j-1} \sum_{i=k+1}^{j} h\left(u_{i}^{n+1}-u_{i}^{n}-w_{i}^{n+1}+w_{i}^{n}\right)
\end{aligned}
$$




$$
\Rightarrow\left|q_{j}^{n+1}-q_{j}^{n}\right| \leqslant 2 \sum_{i=1}^{J} h\left|u_{i}^{n+1}-u_{i}^{n}\right|+2 \sum_{i=1}^{J} h\left|w_{i}^{n+1}-w_{i}^{n}\right| .
$$

Using (6.36), (6.38) and (6.39) gives the required result.

Before we prove the main result of this section we introduce some notation and two useful lemmas.

Let $g^{n} \in X$, for a function space $X$ where $g^{n}$ might be a finite element function. We define,

$$
\begin{array}{ll}
g_{\Delta t}(t):=g^{n} & t \in[n \Delta t,(n+1) \Delta t), \\
g_{\Delta t}^{+}(t):=g^{n+1} & t \in(n \Delta t,(n+1) \Delta t] .
\end{array}
$$

THEOREM 6.1 There exists functions $\{w, u\} \in C\left([0, T] ; L^{1}(\Omega)\right) \cap L^{\infty}(0, T ; B V(\Omega)) \times$ $C\left([0, T] ; H^{1}(\Omega)\right)$ and subsequences such that

$$
\begin{array}{cl}
u_{h, \Delta t} \rightarrow u & \text { strongly in } L^{2}\left([0, T] ; H^{1}(\Omega)\right), \\
f\left(\left(u_{h, \Delta t}\right)_{x}\right) \rightarrow f\left(u_{x}\right) & \text { strongly in } L^{2}\left([0, T] ; L^{2}(\Omega)\right), \\
f^{\prime}\left(\left(u_{h, \Delta t}\right)_{x}\right) \rightarrow f^{\prime}\left(u_{x}\right) & \text { strongly in } L^{2}\left([0, T] ; L^{2}(\Omega)\right), \\
\hat{w}_{h, \Delta t}(t) \rightarrow w(t) & \text { weakly in } L^{2}\left(\Omega_{\mathrm{T}}\right), \\
\hat{w}_{h, \Delta t} \rightarrow w & \text { strongly in } L^{1}\left(\Omega_{\mathrm{T}}\right),
\end{array}
$$

where $\hat{w}_{h, \Delta t}(t)-w_{\mathrm{b}}=\hat{w}_{h}^{n}-w_{\mathrm{b}} \in W_{h}^{0}$ and $u_{h, \Delta t}(t)-u_{\mathrm{b}}=u_{h}^{n}-u_{\mathrm{b}} \in S_{h}^{0}$ for all $t \in[n \Delta t,(n+1) \Delta t)$ and $\{w, u\}$ is the unique solution of (1.39), (1.40) and (1.41).

Proof. Using (6.11), (6.14), (6.16), (6.30) and (6.36) and applying the techniques used in the proof of Lemma 6.1 [9] we obtain (6.42)-(6.46). Furthermore, proving that $\{w, u\}$ satisfies (1.39) and (1.40) follows using a straightforward generalization of the proof of Theorem 6.1 in [9]. We now prove that $\{w, u\}$ satisfies the entropy inequality (1.41). To this end we note that for any $\phi \in C^{\infty}\left(\Omega_{\mathrm{T}}\right)$, the mesh functions $\phi_{h, \Delta t}(t)$ and $\delta_{t}\left(\phi_{h, \Delta t}\right)(t)$ defined on $[0, T)$ by

$$
\begin{aligned}
\phi_{h, \Delta t}(t) & =\pi^{h}(\phi(n \Delta t)) \equiv \phi_{h}^{n}, \quad \forall t \in[n \Delta t,(n+1) \Delta t) \\
\delta_{t}\left(\phi_{h, \Delta t}\right)(t) & =\left(\phi_{h}^{n+1}-\phi_{h}^{n}\right) / \Delta t, \quad \forall t \in[n \Delta t,(n+1) \Delta t)
\end{aligned}
$$

have the approximation properties

$$
\begin{array}{ccl}
t=n \Delta t, & \pi^{h} \phi(t) \rightarrow \phi(t) & \text { strongly in } H_{0}^{1}(\Omega) \\
\phi_{h, \Delta t} \rightarrow \phi & \text { strongly in } L^{2}\left(0, T ; H_{0}^{1}(\Omega)\right) \\
\delta_{t}\left(\phi_{h, \Delta t}\right) \rightarrow \phi_{t} & \text { strongly in } L^{2}\left(0, T ; H_{0}^{1}(\Omega)\right) .
\end{array}
$$

Rewriting (6.10) gives

$$
h\left(w_{j}^{n+1}-w_{j}^{n}\right)=-\Delta t\left(w_{j j+1}^{n+1} f_{j}^{n}-w_{j j-1}^{n+1} f_{j-1}^{n}\right) \quad \forall j \in[1, J-1],
$$

where

$$
w_{j j+1}^{n+1}= \begin{cases}w_{j+1}^{n+1} & \text { if } f_{j}^{n}>0 \\ w_{j}^{n+1} & \text { if } f_{j}^{n}<0\end{cases}
$$


Multiplying (6.48) by $\left(w_{j}^{n+1}-w_{\mathrm{b}}\right)$, noting that $2(a-b) a=(a-b)^{2}+a^{2}-b^{2}$ and applying some algebraic manipulations, gives the following for all $j \in[1, J-1]$

$$
\begin{aligned}
h\left(\left(w_{j}^{n+1}-w_{\mathrm{b}}\right)^{2}-\left(w_{j}^{n}-w_{\mathrm{b}}\right)^{2}\right) \leqslant & \left(w_{j+1}^{n+1}-w_{\mathrm{b}}\right)^{2}\left[f_{j}^{n}\right]_{+}+\left(w_{j}^{n+1}-w_{\mathrm{b}}\right)^{2}\left[f_{j}^{n}\right]_{-} \\
& -\left(w_{j}^{n+1}-w_{\mathrm{b}}\right)^{2}\left[f_{j-1}^{n}\right]_{+}-\left(w_{j-1}^{n+1}-w_{\mathrm{b}}\right)^{2}\left[f_{j-1}^{n}\right]_{-} \\
& +2 \Delta t w_{j}^{n+1}\left(w_{j}^{n+1}-w_{\mathrm{b}}\right)\left(f_{j}^{n}-f_{j-1}^{n}\right) \\
& -\Delta t\left(w_{j}^{n+1}-w_{\mathrm{b}}\right)^{2}\left(f_{j}^{n}-f_{j-1}^{n}\right) .
\end{aligned}
$$

Noting that $f_{j+1}^{n}-f_{j}^{n}=f\left[q_{j+1}^{n}, q_{j}^{n}\right]\left(q_{j+1}^{n}-q_{j}^{n}\right)$, multiplying the above inequality by $\eta_{j}^{n}$ (where $\eta \equiv \eta_{I}(n \Delta t)$ and $\eta \in C^{\infty}\left(\Omega_{\mathrm{T}}\right)$, with $\eta_{0}^{n} \neq 0, \eta_{J}^{n} \neq 0, \eta_{j}^{0}=\eta_{j}^{N-1}=0$ for all $\left.j \in[0, J]\right)$ and summing from $j=1$ to $J-1$ gives

$$
\begin{aligned}
\sum_{j=1}^{J-1} h\left(\left(w_{j}^{n+1}-w_{\mathrm{b}}\right)^{2}-\right. & \left.\left(w_{j}^{n}-w_{\mathrm{b}}\right)^{2}\right) \eta_{j}^{n} \\
\leqslant & \Delta t \sum_{j=0}^{J-1}\left(\left(w_{j+1}^{n+1}-w_{\mathrm{b}}\right)^{2}\left[f_{j}^{n}\right]_{+}-\left(w_{j}^{n+1}-w_{\mathrm{b}}\right)^{2}\left[f_{j}^{n}\right]_{-}\right)\left(\eta_{j}^{n}-\eta_{j+1}^{n}\right) \\
& +\Delta t\left(\left(w_{J}^{n+1}-w_{\mathrm{b}}\right)^{2}\left[f_{J-1}^{n}\right]_{+}-\left(w_{J-1}^{n+1}-w_{\mathrm{b}}\right)^{2}\left[f_{J-1}^{n}\right]_{-}\right) \eta_{J}^{n} \\
& -\Delta t\left(\left(w_{1}^{n+1}-w_{\mathrm{b}}\right)^{2}\left[f_{0}^{n}\right]_{+}-\left(w_{0}^{n+1}-w_{\mathrm{b}}\right)^{2}\left[f_{0}^{n}\right]_{-}\right) \eta_{0}^{n} \\
& +2 h \Delta t \sum_{j=1}^{J-1}\left(u_{j}^{n}-w_{j}^{n}\right) w_{j}^{n+1}\left(w_{j}^{n+1}-w_{\mathrm{b}}\right) \eta_{j}^{n} f\left[q_{j}^{n}, q_{j-1}^{n}\right] \\
& -h \Delta t \sum_{j=1}^{J-1}\left(u_{j}^{n}-w_{j}^{n}\right)\left(w_{j}^{n+1}-w_{\mathrm{b}}\right)^{2} \eta_{j}^{n} f\left[q_{j}^{n}, q_{j-1}^{n}\right] .
\end{aligned}
$$

Since $w_{0}^{n}=w_{J}^{n}=w_{\mathrm{b}}$ using some simple algebraic manipulations, and noting that $w_{h}^{n}, u_{h}^{n}$ and $f_{h}^{n}$ are bounded in $L^{\infty}(\Omega)$, we have

$$
\begin{aligned}
\sum_{j=1}^{J-1} h\left(\left(w_{j}^{n+1}-\right.\right. & \left.\left.w_{\mathrm{b}}\right)^{2}-\left(w_{j}^{n}-w_{\mathrm{b}}\right)^{2}\right) \eta_{j}^{n} \\
\leqslant & \frac{\Delta t}{2} \sum_{j=0}^{J-1}\left(\left(w_{j+1}^{n+1}-w_{\mathrm{b}}\right)^{2}+\left(w_{j}^{n+1}-w_{\mathrm{b}}\right)^{2}\right) f_{j}^{n}\left(\eta_{j}^{n}-\eta_{j+1}^{n}\right) \\
& +\frac{\Delta t}{2} \sum_{j=0}^{J-1}\left(\left(w_{j+1}^{n+1}-w_{\mathrm{b}}\right)^{2}-\left(w_{j}^{n}-w_{\mathrm{b}}\right)^{2}\right)\left(\left[f_{j}^{n}\right]_{+}-\left[f_{j}^{n}\right]-\right)\left(\eta_{j}^{n}-\eta_{j+1}^{n}\right) \\
& +h \Delta t \sum_{j=1}^{J-1}\left(2 w_{j}^{n+1}\left(w_{j}^{n+1}-w_{\mathrm{b}}\right)-\left(w_{j}^{n}-w_{\mathrm{b}}\right)^{2}\right)\left(u_{j}^{n}-w_{j}^{n}\right) f\left[q_{j}^{n}, q_{j-1}^{n}\right] \eta_{j}^{n} \\
= & \frac{\Delta t}{2} \sum_{j=0}^{J-1}\left(\left(w_{j+1}^{n+1}-w_{\mathrm{b}}\right)^{2}+\left(w_{j}^{n+1}-w_{\mathrm{b}}\right)^{2}\right) f_{j}^{n}\left(\eta_{j}^{n}-\eta_{j+1}^{n}\right)
\end{aligned}
$$




$$
\begin{aligned}
& \left.+\Delta t \sum_{j=1}^{J-1} h\left(2 w_{j}^{n+1}\left(w_{j}^{n+1}-w_{\mathrm{b}}\right)-\left(w_{j}^{n}-w_{\mathrm{b}}\right)^{2}\right)\left(u_{j}^{n}-w_{j}^{n}\right) f\left[q_{j}^{n}, q_{j-1}^{n}\right] \eta_{j}^{n}\right) \\
& +\frac{\Delta t}{2} \sum_{j=0}^{J-1}\left(w_{j+1}^{n+1}-w_{j}^{n}\right)\left(\left(w_{j+1}^{n+1}+w_{j}^{n}\right)-2 w_{\mathrm{b}}\right)\left(\left[f_{j}^{n}\right]_{+}-\left[f_{j}^{n}\right]_{-}\right)\left(\eta_{j}^{n}-\eta_{j+1}^{n}\right) \\
& \leqslant \sum_{j=0}^{J-1}\left(\left(w_{j+1}^{n+1}-w_{\mathrm{b}}\right)^{2}+\left(w_{j}^{n+1}-w_{\mathrm{b}}\right)^{2}\right) f_{j}^{n}\left(\eta_{j}^{n}-\eta_{j+1}^{n}\right) \\
& \left.+\Delta t \sum_{j=1}^{J-1} h\left(2 w_{j}^{n+1}\left(w_{j}^{n+1}-w_{\mathrm{b}}\right)-\left(w_{j}^{n}-w_{\mathrm{b}}\right)^{2}\right)\left(u_{j}^{n}-w_{j}^{n}\right) f\left[q_{j}^{n}, q_{j-1}^{n}\right] \eta_{j}^{n}\right) \\
& +C \Delta t \sum_{j=0}^{J-1}\left|w_{j+1}^{n+1}-w_{j}^{n}\right|\left|\eta_{j}^{n}-\eta_{j+1}^{n}\right| .
\end{aligned}
$$

Since $f\left[q_{j}, q_{j-1}\right]=\left(f\left(q_{j}\right)-f\left(q_{j-1}\right)\right) /\left(q_{j}-q_{j-1}\right)$ we set

$$
f\left[q_{j}^{n}, q_{j-1}^{n}\right]=f^{\prime}\left(q_{j}^{n}\right)+\frac{1}{2} f^{\prime \prime}(\xi)\left(q_{j-1}^{n}-q_{j}^{n}\right)=f^{\prime}\left(q_{j}^{n}\right)+\frac{h}{2} f^{\prime \prime}(\xi)\left(w_{j}^{n}-u_{j}^{n}\right),
$$

where $\xi \in\left(q_{j-1}^{n}, q_{j}^{n}\right)$, and from the boundedness of $f^{\prime \prime}$ we conclude that

$$
\begin{aligned}
\sum_{j=1}^{J-1} h\left(\left(w_{j}^{n+1}-\right.\right. & \left.\left.w_{\mathrm{b}}\right)^{2}-\left(w_{j}^{n}-w_{\mathrm{b}}\right)^{2}\right) \eta_{j}^{n} \\
\leqslant & \int_{0}^{1}-\left(w_{h}^{n+1}-w_{\mathrm{b}}\right)^{2} f\left(\left(u_{h}^{n}\right)_{x}\right)\left(\eta_{h}^{n}\right)_{x} \mathrm{~d} x \\
& +\left(f^{\prime}\left(\left(u_{h}^{n}\right)_{x}\right)\right)\left(u_{h}^{n}-w_{h}^{n}\right)\left(2 w_{h}^{n+1}\left(w_{h}^{n+1}-w_{\mathrm{b}}\right)-\left(\left(w_{h}^{n+1}-w_{\mathrm{b}}\right)^{2}\right), \eta_{h}^{n}\right)_{h} \\
& -C \Delta t \sum_{j=1}^{J-1} h^{2}\left|w_{j}^{n+1}\left(w_{j}^{n+1}-w_{\mathrm{b}}\right)-\left(w_{j}^{n}-w_{\mathrm{b}}\right)^{2}\right|\left(u_{j}^{n}-w_{j}^{n}\right)^{2} \\
& +C \Delta t \sum_{j=0}^{J-1}\left|w_{j+1}^{n+1}-w_{j}^{n}\right|\left|\eta_{j}^{n}-\eta_{j+1}^{n}\right| .
\end{aligned}
$$

Summing the above inequality from $n=0$ to $n=N-1$ and noting that $\eta_{j}^{0}=\eta_{j}^{N}=0$ for all $j \in[0, J]$ and that $\left|\eta_{j+1}^{n}-\eta_{j}^{n}\right| \leqslant C h\left(\right.$ since $\left.\eta \in C^{\infty}\left(\Omega_{\mathrm{T}}\right)\right)$ we have

$$
\begin{aligned}
& \left.-\int_{0}^{T}\left(w_{h, \Delta t}^{+}(t) w_{\mathrm{b}}\right)^{2}, \delta_{t} \eta_{h, \Delta t}(t)\right)_{h} \mathrm{~d} t \\
& \leqslant-\int_{0}^{T} \int_{0}^{1}\left(w_{h, \Delta t}^{+}(t)-w_{\mathrm{b}}\right)^{2} f\left(\left(u_{h, \Delta t}\right)_{x}(t)\right)\left(\eta_{h, \Delta t}\right)_{x}(t) \mathrm{d} x \mathrm{~d} t \\
& \quad+2 \int_{0}^{T}\left(f^{\prime}\left(\left(u_{h, \Delta t}\right)_{x}(t)\right) w_{h, \Delta t}^{+}(t)\left(w_{h, \Delta t}^{+}(t)-w_{\mathrm{b}}\right)\left(u_{h, \Delta t}(t)-w_{h, \Delta t}(t)\right), \eta_{h, \Delta t}(t)\right)_{h} \mathrm{~d} t
\end{aligned}
$$




$$
\begin{aligned}
& -\int_{0}^{T}\left(f^{\prime}\left(\left(u_{h, \Delta t}\right)_{x}(t)\right)\left(w_{h, \Delta t}^{+}(t)-w_{\mathrm{b}}\right)^{2}\left(u_{h, \Delta t}(t)-w_{h, \Delta t}(t)\right), \eta_{h, \Delta t}(t)\right)_{h} \mathrm{~d} t \\
& +C h+C h \Delta t \sum_{n=0}^{N-1} \sum_{j=1}^{J-1}\left|w_{j+1}^{n+1}-w_{j}^{n+1}\right|\left|\eta_{j}^{n}-\eta_{j+1}^{n}\right| .
\end{aligned}
$$

Noting that $\eta \in C^{\infty}\left(\Omega_{\mathrm{T}}\right)$ it follows that $\eta_{h, \Delta t}$ and $\eta_{h, \Delta t}^{\prime}$, satisfy the convergence properties (6.47), thus letting $h$ and $\Delta t$ tend to zero in (6.51) and using the strong convergence properties of $f\left(u_{h, \Delta t}\right)$, $f^{\prime}\left(u_{h, \Delta t}\right), w_{h, \Delta t}, \eta_{h, \Delta t}$ and $\delta_{t} \eta_{h, \Delta t},(6.42),(6.46)$ and (6.47), we obtain (1.41). Thus $\{w, u\}$ satisfies (1.41) and from this, [20], we can conclude that $w$ also satisfies $w=w_{\mathrm{b}}$ on all inflow boundary sections and hence $\{w, u\}$ is the unique solution of (1.39), (1.40) and (1.41). It also follows that the whole sequences $u_{h, \Delta t}$ and $w_{h, \Delta t}$ converge.

\section{Steady-state solutions of the discretization}

Throughout this section we denote the steady-state solutions of $w_{h, \Delta t}$ and $u_{h, \Delta t}$ by $\tilde{w}_{h}$ and $\tilde{u}_{h}$, respectively.

LEMMA 7.1 All points $\left\{\tilde{w}_{h}, \tilde{u}_{h}\right\} \in L^{\infty}(\Omega) \times H^{1}(\Omega)$ of the $\omega$-limit set:

$$
\omega\left(w_{0}^{h}\right)=\left\{\left(\tilde{w}_{h}, \tilde{u}_{h}\right) \in W_{h}^{w_{\mathrm{b}}} \times S_{h}: \exists\left\{n_{k}\right\} \text { s.t. } w_{h}^{n_{k}} \rightarrow \tilde{w}_{h}, u_{h} \rightarrow \tilde{u}_{h} \text { as } n_{k} \rightarrow \infty\right\},
$$

are steady-state solutions which satisfy

$$
\begin{array}{ll}
\tilde{w}_{j+1}\left[\tilde{f}_{j}\right]_{-}^{2}+\tilde{w}_{j}\left[\tilde{f}_{j}\right]_{+}^{2}=0 & \forall j \in[0, J-1], \\
\lambda^{2}\left(\tilde{q}_{j}-\tilde{q}_{j-1}\right)=\tilde{u}_{j}-\tilde{w}_{j} & \forall j \in[1, J-1],
\end{array}
$$

and

$$
\tilde{u}_{0}=\tilde{u}_{J}=u_{\mathrm{b}}
$$

where $\tilde{q}_{j}=\frac{\left(\tilde{u}_{j+1}-\tilde{u}_{j}\right)}{h}$ and $\tilde{f}_{j}^{n}=f\left(\tilde{q}_{j}\right)$.

Proof. From Lemma 6.1 it follows that there exist functions $\left\{\tilde{w}_{h}, \tilde{u}_{h}\right\}$ and a sequence $n_{k}$, such that as $n_{k} \rightarrow \infty$

$$
w_{h}^{n_{k}} \rightarrow \tilde{w}_{h} \quad \text { and } \quad u_{h}^{n_{k}} \rightarrow \tilde{u}_{h},
$$

and hence $\omega\left(w_{h}^{0}\right)$ is non-empty. Summing (6.26) from $n=0$ to $N-1$ we have

$$
E_{h}\left(u_{h}^{N}-u_{\mathrm{b}}\right)+\left(1 / \mu-\frac{\varepsilon}{2 \lambda^{2}}\right) \sum_{n=0}^{N-1} \Delta t \sum_{j=0}^{J-1}\left(w_{j+1}^{n+1}\left[f_{j}^{n}\right]_{+}^{2}+w_{j}^{n+1}\left[f_{j}^{n}\right]_{-}^{2}\right) \leqslant E_{h}\left(u_{h}^{0}-u_{\mathrm{b}}\right) .
$$

Since $u_{h}^{0}$ is the interpolant of $u^{0} \in H^{1}(\Omega)$ and the discrete $L^{2}$-norm is equivalent to the $L^{2}$-norm, it follows that

$$
\sum_{n=0}^{N-1} \Delta t \sum_{j=0}^{J-1}\left(w_{j+1}^{n+1}\left[f_{j}^{n}\right]_{+}^{2}+w_{j}^{n+1}\left[f_{j}^{n}\right]_{-}^{2}\right) \leqslant C
$$


Since (7.5) holds for all $N>0$ it follows that

$$
\lim _{n_{k} \rightarrow \infty}\left(w_{j+1}^{n+1}\left[f_{j}^{n}\right]_{+}^{2}+w_{j}^{n+1}\left[f_{j}^{n}\right]_{-}^{2}\right)=0,
$$

and from (6.10) and (7.6) we can conclude that

$$
\lim _{n_{k} \rightarrow \infty} w_{j}^{n_{k}+1}=\lim _{n_{k} \rightarrow \infty} w_{j}^{n_{k}}=\tilde{w}_{j}
$$

Since (6.9) and (6.12) hold for all $n \geqslant 0$, from (7.7) we can conclude that $\left\{\tilde{w}_{h}, \tilde{u}_{h}\right\}$ is a steady-state solution satisfying (7.1)-(7.3).

\section{Numerical computations}

In this section we display some one- and two-dimensional computations obtained using the numerical scheme (6.7)-(6.12) with $f(r):=\operatorname{sgn}(r)\left[|r|-J_{p}\right]_{+}$, and an equivalent two-dimensional scheme for a uniform right-angled triangular mesh. We note that although this choice of $f$ coincides with the pinning function defined in [4], it does not satisfy (6.11). However, the numerical results produced using this value of $f$ are consistent with results obtained using 'smoother' versions of $f$. For the one-dimensional results we set $\Omega=(0,1)$ and $h=0.01$, while for the two-dimensional results we set $\Omega=(0,1) \times(0.1)$ and $h=0.01$, with $\Delta t$ satisfying (6.23) in both cases. We begin with a brief description of the two-dimensional scheme followed by an introduction to the computational results displayed.

\section{Two-dimensional numerical scheme}

We now define a numerical approximation of the two-dimensional model (1.15)-(1.19), with $J_{p}=$ $J_{n}$ and $w(x, t)=w_{\mathrm{b}}$ for all $(x, t) \in \partial \Omega_{\mathrm{T}}$. We discretize (1.16) and (1.18) using a standard finite element approximation,

$$
\begin{gathered}
a_{h}\left(u_{h}^{n}, \chi\right)=\left(w_{h}^{n}, \chi\right)_{h} \quad \forall \chi \in S_{h}^{0}, \forall n \geqslant 0, \\
u_{h}^{n}-u_{\mathrm{b}} \in S_{h}^{0}, \forall n \geqslant 0,
\end{gathered}
$$

and we discretize (1.15) using a semi-implicit upwinding finite volume scheme [14]. To implement the finite volume scheme we first create a dual mesh by perpendicularly bisecting each edge of our triangulation, giving rise to dual cells denoted by $V_{j}$ associated to each node $x_{j}$ of the original triangulation. To approximate (1.15) we integrate it over each interior dual cell $V_{j}=V_{j 1} \cup V_{j 2} \cup$ $V j 3 \cup V_{j 4} \cup V_{j 5} \cup V_{j 6}$ (see Fig. 1), to obtain

$$
\int_{V_{j}} w_{t} \mathrm{~d} x=\int_{V_{j}} \nabla \cdot(w \mathbf{v}) \mathrm{d} x=\int_{\partial V_{j}} w \mathbf{v} \cdot \mathbf{n} \mathrm{d} S=-\int_{\partial V_{j}} w f(|\nabla u|) \frac{\partial u}{\partial \mathbf{n}} \mathrm{d} S .
$$

Substituting $w_{h} \in W_{h}$ and $u_{h} \in S_{h}$ for $w$ and $u$ in (8.3) we have

$$
\int_{V_{j}}\left(w_{h}\right)_{t} \mathrm{~d} x=-\int_{\partial V_{j}} w_{h}\left(f\left(\left|\nabla u_{h}\right|\right) \frac{\partial u_{h}}{\partial \mathbf{n}} \mathrm{d} S .\right.
$$




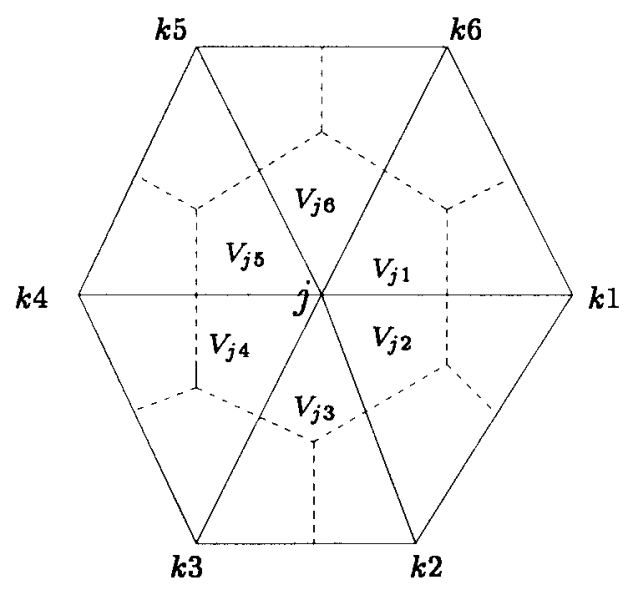

FIG. 1. Dual mesh.

Setting

$$
\begin{aligned}
& A=\partial V_{j 1} \cap \partial V_{k 1}, \quad B=\partial V_{j 2} \cap \partial V_{k 1}, \quad C=\partial V_{j 2} \cap \partial V_{k 2}, \\
& D=\partial V_{j 3} \cap \partial V_{k 2}, \quad E=\partial V_{j 3} \cap \partial V_{k 3}, \quad F=\partial V_{j 4} \cap \partial V_{k 3} \text {, } \\
& G=\partial V_{j 4} \cap \partial V_{k 4}, \quad H=\partial V_{j 5} \cap \partial V_{k 4}, \quad I=\partial V_{j 5} \cap \partial V_{k 5} \text {, } \\
& J=\partial V_{j 6} \cap \partial V_{k 5}, \quad K=\partial V_{j 6} \cap \partial V_{k 6}, \quad L=\partial V_{j 1} \cap \partial V_{k 6},
\end{aligned}
$$

where $V_{j 1}, V_{j 2}$, etc are defined in Fig. 1, and denoting the lengths of $A$ through to $L$, by $A^{\prime}$ through to $L^{\prime}$, and the length of the edge joining nodes $j$ and $k$ by $h_{j k}$ we have

$$
\begin{array}{llrl}
\int_{A+B} \frac{\partial u}{\partial \mathbf{n}} \mathrm{d} S & =\left(A^{\prime}+B^{\prime}\right)\left(u_{k 1}^{n}-u_{j}^{n}\right) / h_{j k}, & & \int_{C+D} \frac{\partial u}{\partial \mathbf{n}} \mathrm{d} S=\left(C^{\prime}+D^{\prime}\right)\left(u_{k 2}^{n}-u_{j}^{n}\right) / h_{j k}, \\
\int_{E+F} \frac{\partial u}{\partial \mathbf{n}} \mathrm{d} S=\left(E^{\prime}+F^{\prime}\right)\left(u_{k 3}^{n}-u_{j}^{n}\right) / h_{j k}, & \int_{G+H} \frac{\partial u}{\partial \mathbf{n}} \mathrm{d} S=\left(G^{\prime}+H^{\prime}\right)\left(u_{k 4}^{n}-u_{j}^{n}\right) / h_{j k}, \\
\int_{I+J} \frac{\partial u}{\partial \mathbf{n}} \mathrm{d} S=\left(I^{\prime}+J^{\prime}\right)\left(u_{k 5}^{n}-u_{j}^{n}\right) / h_{j k}, & & \int_{K+A} \frac{\partial u}{\partial \mathbf{n}} \mathrm{d} S=\left(K^{\prime}+A^{\prime}\right)\left(u_{k 6}^{n}-u_{j}^{n}\right) / h_{j k} .
\end{array}
$$

We approximate $\int_{V_{j}} w_{t} \mathrm{~d} x$ with a time difference by $\frac{m_{j}}{\Delta t}\left(w_{j}^{n+1}-w_{j}^{n}\right)$, and we use an upwinding scheme to approximate $w$ in the right-hand side of (8.4), since $f(|\nabla u|) \geqslant 0$ for all $x \in \Omega$ we set our approximate value of $w$ to depend on the sign of $\frac{\partial u}{\partial \mathbf{n}}$ on each section of $\partial V_{j}$; yielding the following for all $n \geqslant 1$ and all interior nodes $j$

$$
\begin{aligned}
m_{j}\left(w_{j}^{n+1}-\right. & \left.w_{j}^{n}\right) / \Delta t= \\
& \left.\left.\left(f\left(A_{j}\right) A^{\prime}+f\left(B_{j}\right) B^{\prime}\right)\left(w_{k 1}^{n+1}\left[u_{k 1}^{n}-u_{j}^{n}\right)\right]_{+}+w_{j}^{n+1}\left[u_{k 1}^{n}-u_{j}^{n}\right)\right]_{-}\right) / h_{j k} \\
& \left.\left.+\left(f\left(C_{j}\right) C^{\prime}+f\left(D_{j}\right) D^{\prime}\right)\left(w_{k 2}^{n+1}\left[u_{k 2}^{n}-u_{j}^{n}\right)\right]_{+}+w_{j}^{n+1}\left[u_{k 2}^{n}-u_{j}^{n}\right)\right]_{-}\right) / h_{j k} \\
& \left.\left.+\left(f\left(E_{j}\right) E^{\prime}+f\left(F_{j}\right) F^{\prime}\right)\left(w_{k 3}^{n+1}\left[u_{k 3}^{n}-u_{j}^{n}\right)\right]_{+}+w_{j}^{n+1}\left[u_{k 3}^{n}-u_{j}^{n}\right)\right]_{-}\right) / h_{j k} \\
& \left.\left.+\left(f\left(G_{j}\right) G^{\prime}+f\left(H_{j}\right) H^{\prime}\right)\left(w_{k 4}^{n+1}\left[u_{k 4}^{n}-u_{j}^{n}\right)\right]_{+}+w_{j}^{n+1}\left[u_{k 4}^{n}-u_{j}^{n}\right)\right]_{-}\right) / h_{j k} \\
& \left.\left.+\left(f\left(I_{j}\right) I^{\prime}+f\left(J_{j}\right) J^{\prime}\right)\left(w_{k 5}^{n+1}\left[u_{k 5}^{n}-u_{j}^{n}\right)\right]_{+}+w_{j}^{n+1}\left[u_{k 5}^{n}-u_{j}^{n}\right)\right]_{-}\right) / h_{j k} \\
& \left.\left.+\left(f\left(K_{j}\right) K^{\prime}+f\left(A_{j}\right) A^{\prime}\right)\left(w_{k 6}^{n+1}\left[u_{k 6}^{n}-u_{j}^{n}\right)\right]_{+}+w_{j}^{n+1}\left[u_{k 6}^{n}-u_{j}^{n}\right)\right]_{-}\right) / h_{j k},
\end{aligned}
$$

where $f\left(A_{j}\right)=f\left(\left|\nabla u_{h}\right|\right)$ evaluated on the edge $A, f\left(B_{j}\right)=f\left(\left|\nabla u_{h}\right|\right)$ evaluated on the edge $B$, etc.

We set $w_{j}^{n}=w_{\mathrm{b}}$ for all $n \geqslant 1$ and all boundary nodes $j$. 

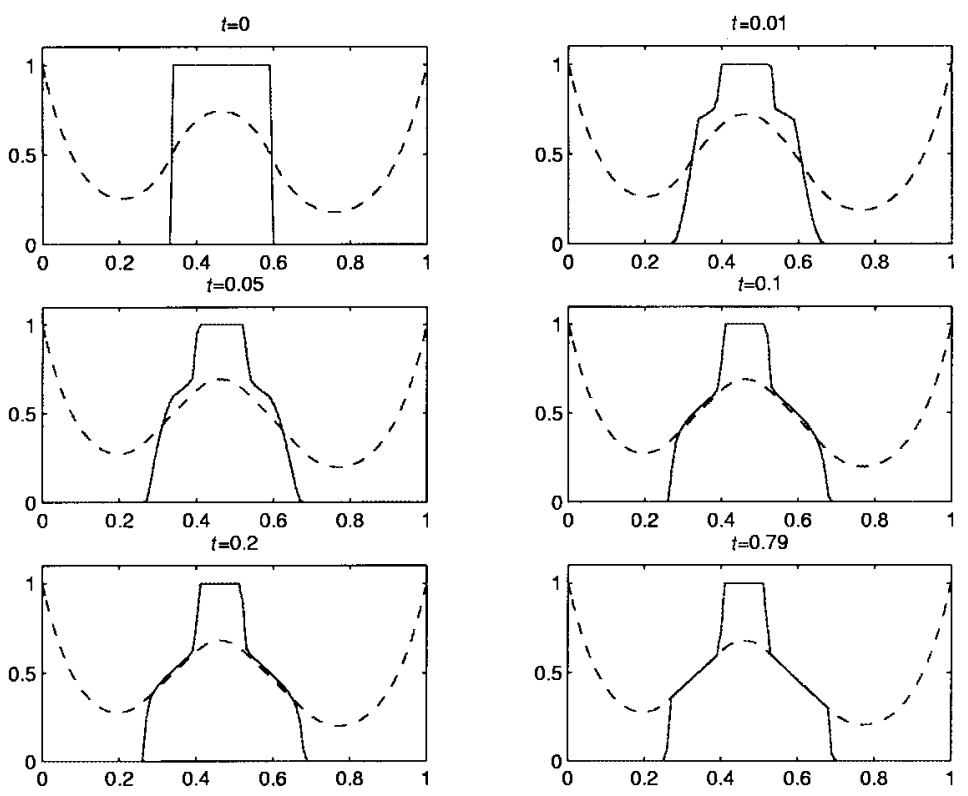

FIG. 2. Evolution with pinning, $J_{p}=2, u_{\mathrm{b}}=1, w_{\mathrm{b}}=0$.
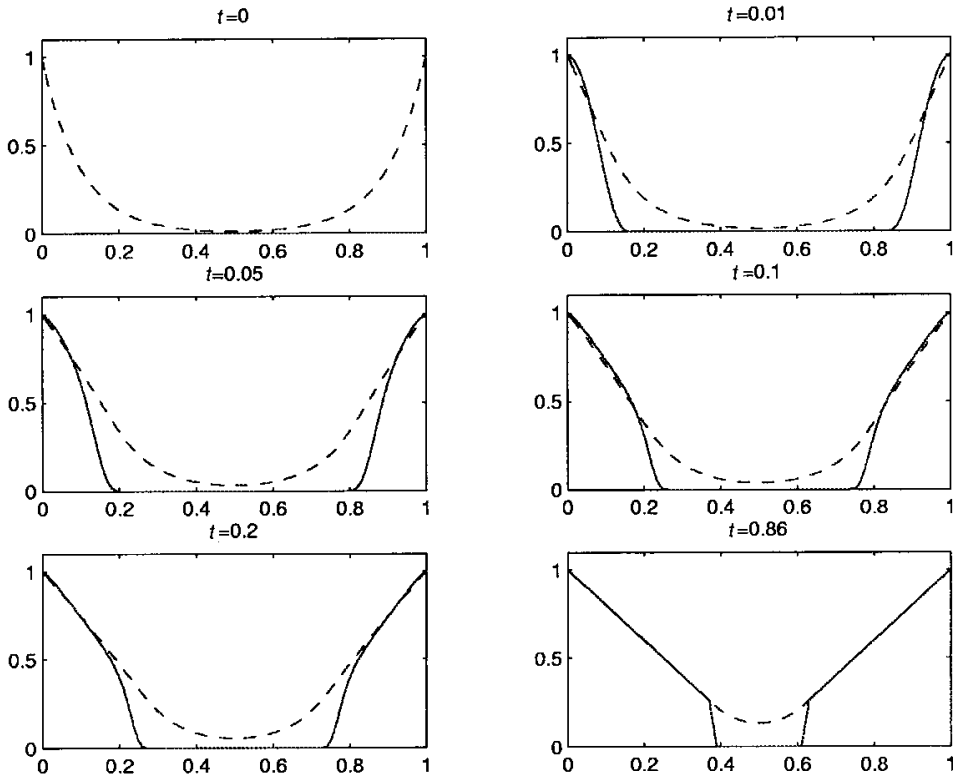

FIG. 3. Evolution with nucleation and pinning, $J_{p}=J_{n}=2, u_{\mathrm{b}}=w_{\mathrm{b}}=1$. 

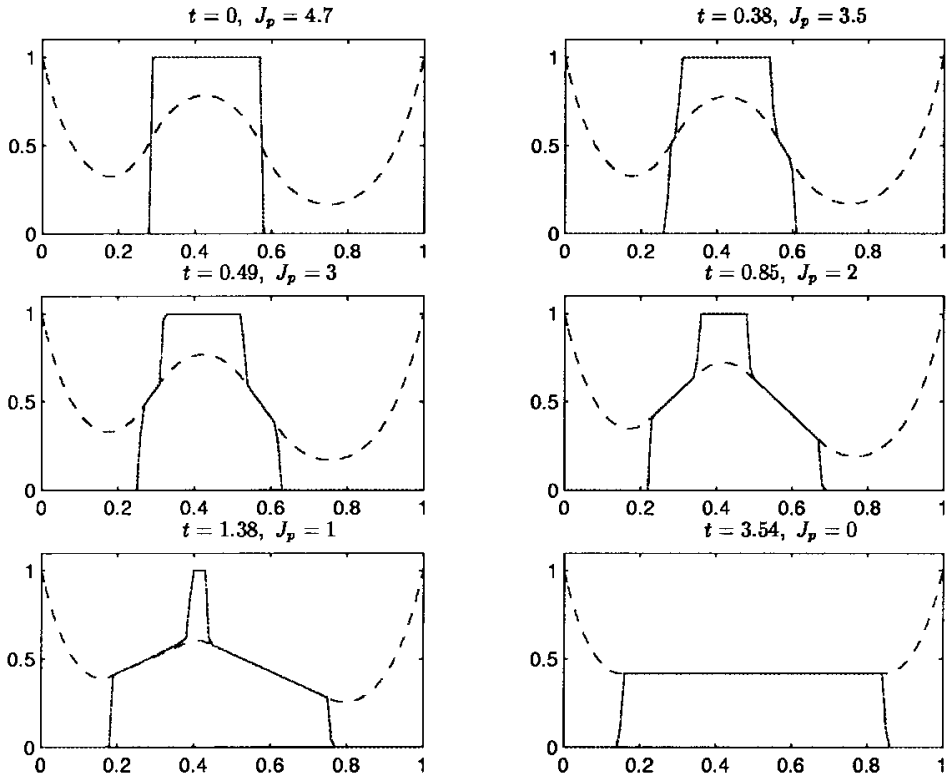

FIG. 4. Steady states with pinning, $u_{\mathrm{b}}=1, w_{\mathrm{b}}=0$.
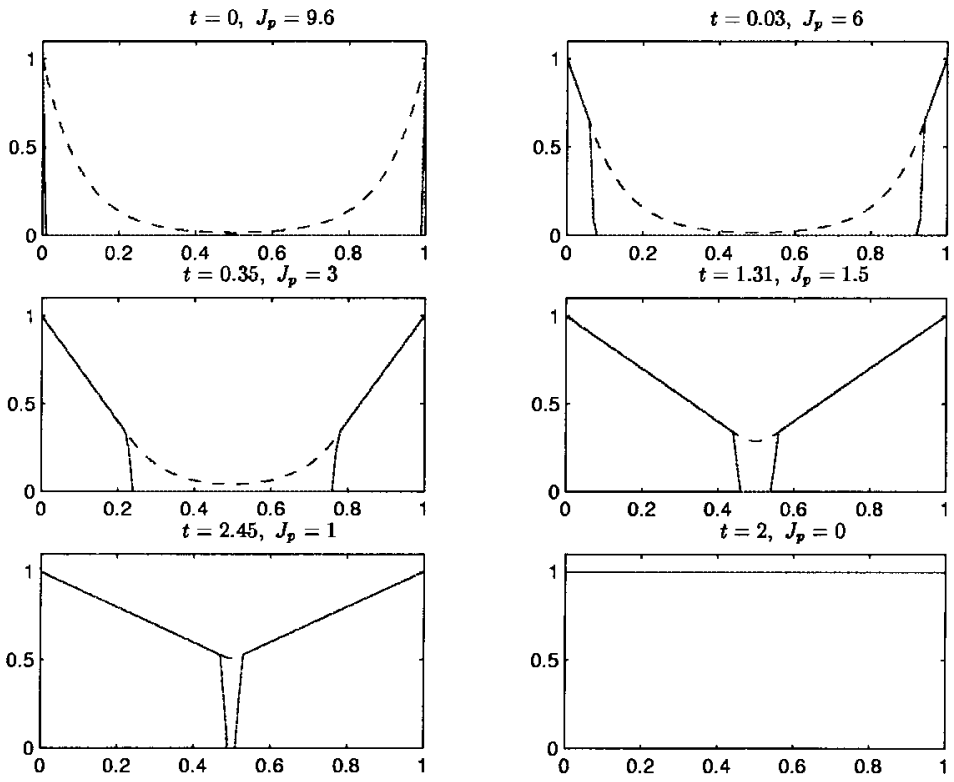

FIG. 5. Steady states with nucleation and pinning, $u_{\mathrm{b}}=w_{\mathrm{b}}=1$. 

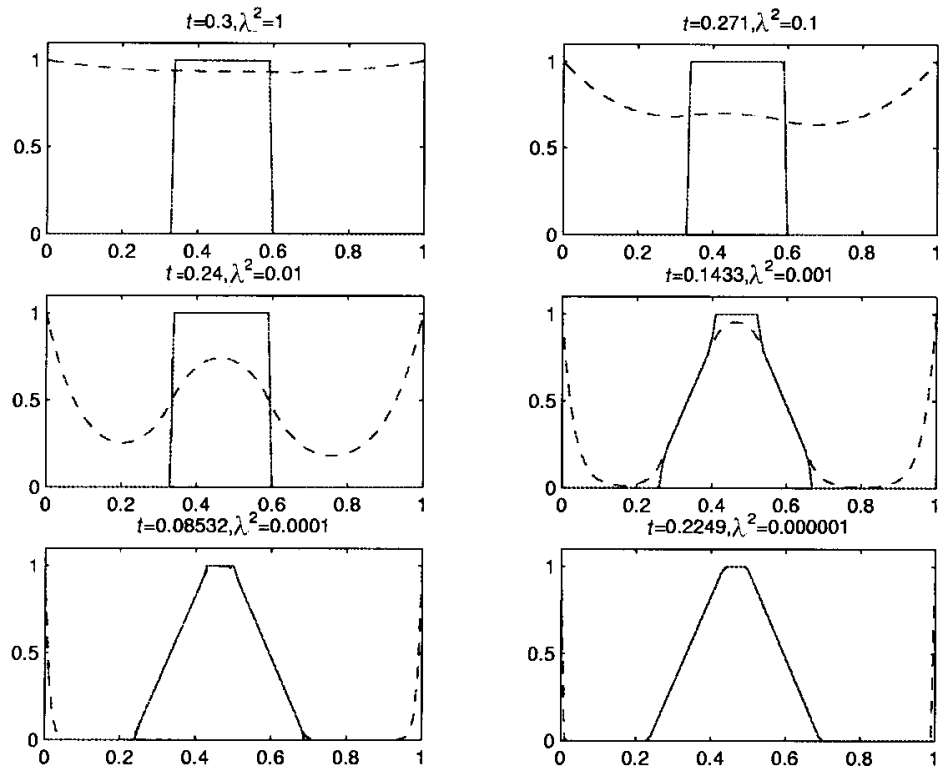

FIG. 6. Steady states with pinning, $J_{p}=2, u_{\mathrm{b}}=1, w_{\mathrm{b}}=0$.
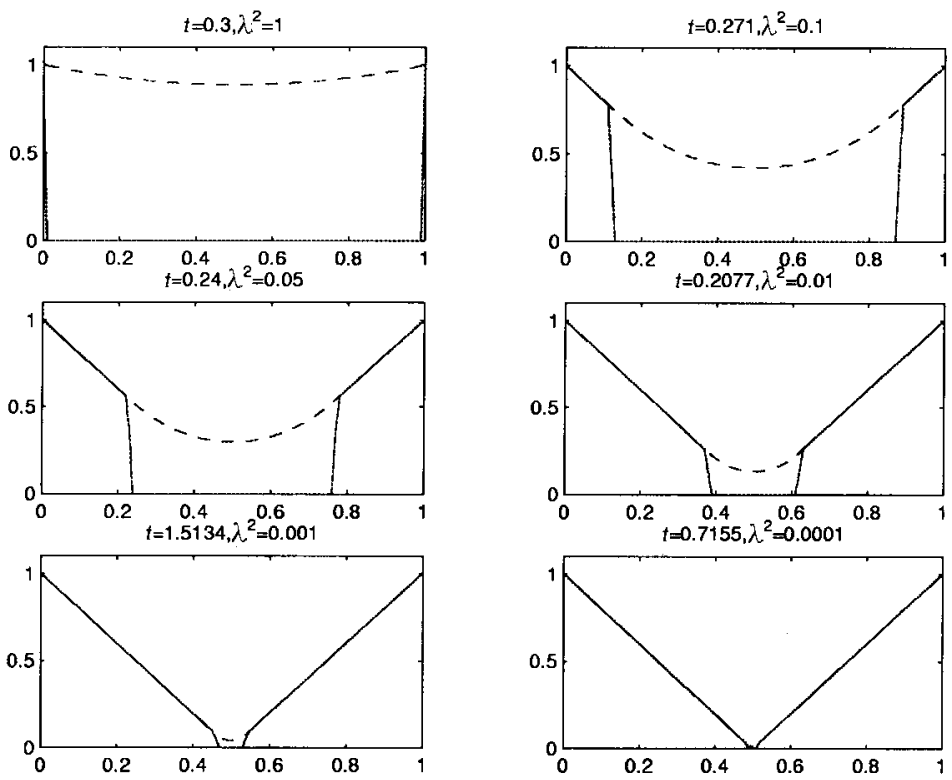

FIG. 7. Steady states with nucleation and pinning, $J_{p}=J_{n}=2, u_{\mathrm{b}}=w_{\mathrm{b}}=1$. 

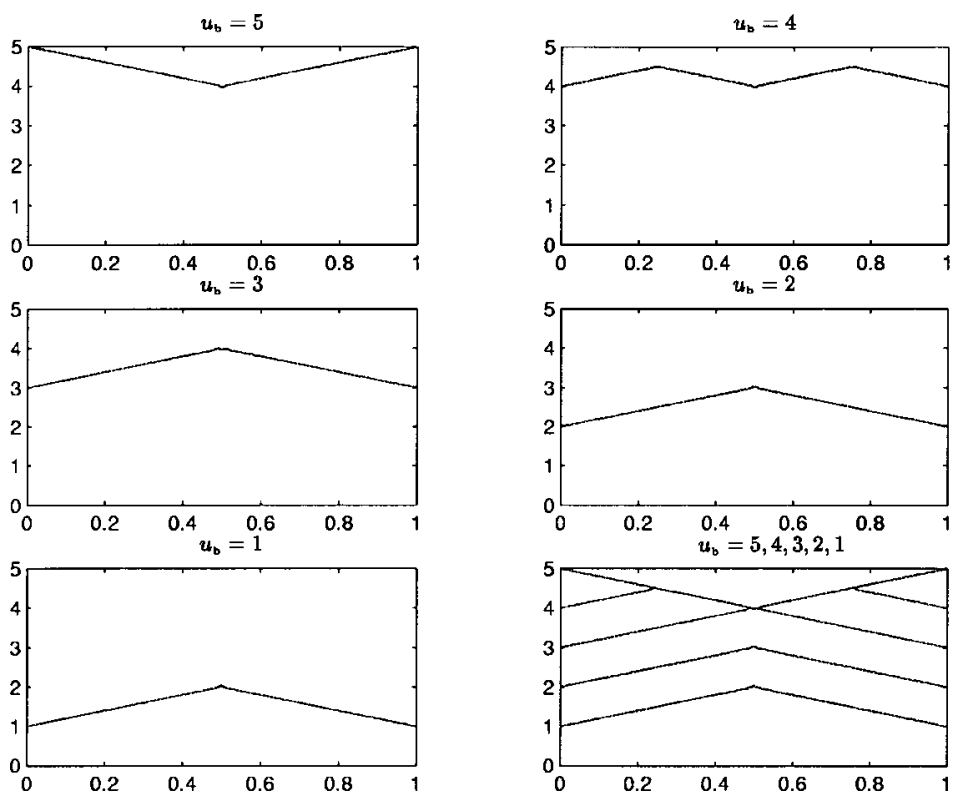

FIG. 8. Bean model simulations, $\lambda^{2}=0.0001, J_{p}=J_{n}=2, w_{\mathrm{b}}=u_{\mathrm{b}}$.
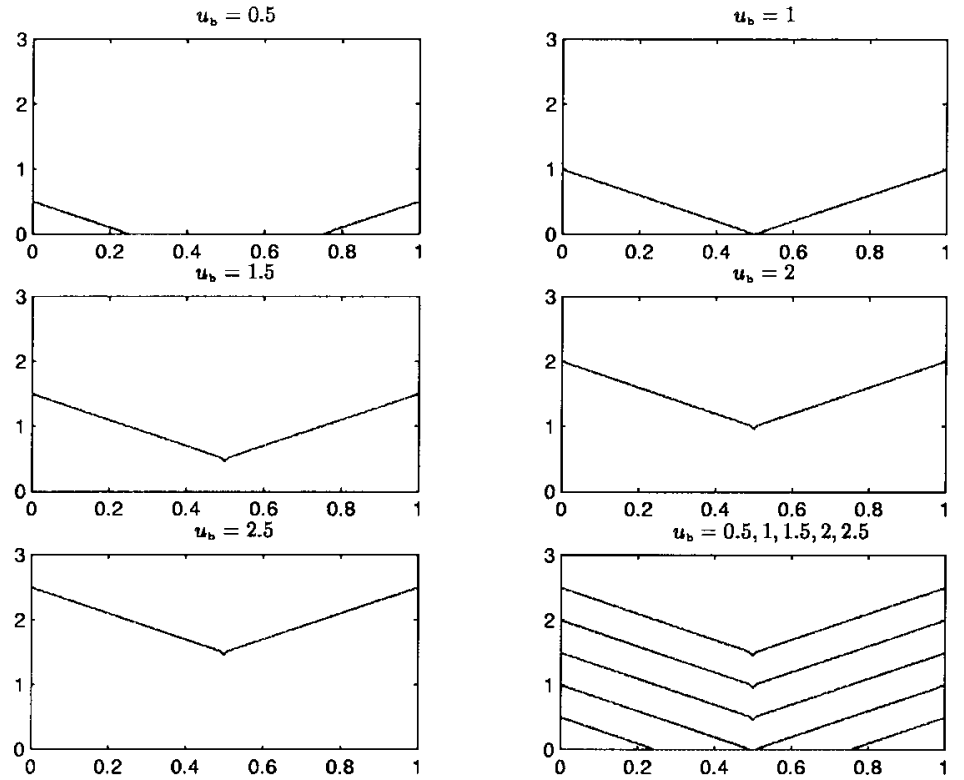

FIG. 9. Bean model simulations, $\lambda^{2}=0.0001, J_{p}=J_{n}=2, w_{\mathrm{b}}=u_{\mathrm{b}}$. 


\section{One-dimensional computations}

The one-dimensional results are displayed in Figs 2-9, in which plots of $w_{h}(t)$ (bold line) and $u_{h}(t)$ (dashed line) are displayed together on one plot, at various times $t$. Figures 2-9 can be divided up into four sets, the first set, Figs 2 and 3, show the evolution of $w_{h}$ and $u_{h}$ from some given initial data (top left-hand subplots) to their steady states $\tilde{w}_{h}$ and $\tilde{u}_{h}$ (bottom right-hand subplots). The second set of results, Figs 4 and 5, each contain six subplots showing the steady states $\tilde{w}_{h}$ and $\tilde{u}_{h}$, obtained using the same initial data but different values of $J_{p}=J_{n}$. The top left-hand subplots in Figs 4 and 5 show $\tilde{w}_{h}$ and $\tilde{u}_{h}$ obtained by taking $J_{p}=J_{n}$ sufficiently large so that all the vorticity is pinned and no vorticity enters the domain, thus in fact these top left-hand subplots are plots of the initial data from which the remaining five steady states have evolved. At the top of each subplot are the values of $J_{p}$ and $J_{n}$ that were used and the time taken for the steady state to be reached. In Figs 2-5 we set $\lambda=0.1$, while in Figs 6 and 7 we display steady-state solutions obtained using varying values of $\lambda$. The top left-hand subplot shows the initial data $w_{h}(0)$ and $u_{h}(0)$ while the three remaining plots show steady states obtained from this data using the same values of $J_{p}=J_{n}, u_{\mathrm{b}}$ and $w_{\mathrm{b}}$ but different values of $\lambda$.

The fourth set of figures, Figs 8 and 9, show the following results; the top left-hand subplot displays the steady-state solution, obtained using no initial vorticity and the value of $u_{\mathrm{b}}$ displayed above the subplot, the top middle subplot depicts the steady-state solution obtained using the steadystate solution of the previous subplot as initial data and a different value of $u_{\mathrm{b}}$. Similarly, the remaining subplots (with the exception of the bottom right-hand subplot) are steady-state solutions obtained using varying $u_{\mathrm{b}}$ 's and the subplot on their left (or on the subplot on the end of the row above) as initial data. The bottom right-hand subplot displays the results of the previous four subplots together in one subplot.

\section{Two-dimensional computations}

The two-dimensional results, Figs 10-17, are displayed in much the same format as the first four one-dimensional figures, except that this time we display contour plots of $w_{h}$ and $u_{h}$ in separate figures which we group together on the same page. The first four two-dimensional figures, Figs 1013, show the evolution of initial data to their steady state, while the remaining figures, Figs 14-17, show the steady-state solutions $\tilde{w}_{h}$ and $\tilde{u}_{h}$ obtained using various values of $J_{p}=J_{n}$. In all the two-dimensional results we have taken $\lambda=0.1$.

\section{Discussion of the numerical results}

Figures 2, 4, 10, 11, 14 and 15 display results obtained for superconducting samples in which flux pinning of vorticity occurs, but no vorticity is nucleated at the boundary (i.e. samples in which $J_{n}$ is very large). From these figures we see that the minimum value of the magnitude of the current $J:=\left|u_{x}\right|$ (which is in fact $J=0$ ) occurs at the centre of the initial blocks of vorticity and as a result the vorticity remains pinned to this site throughout the computation, while the vorticity situated away from the centre of the block spreads out either side of the block. Clearly the greater the value of the critical pinning current $J_{p}$, the less the vorticity is free to move and the less spread out the block becomes.

Figures 3, 5, 12, 13, 16 and 17 display results obtained for superconducting samples initially containing no flux lines. Vorticity enters by nucleation at the boundary and we take the critical 

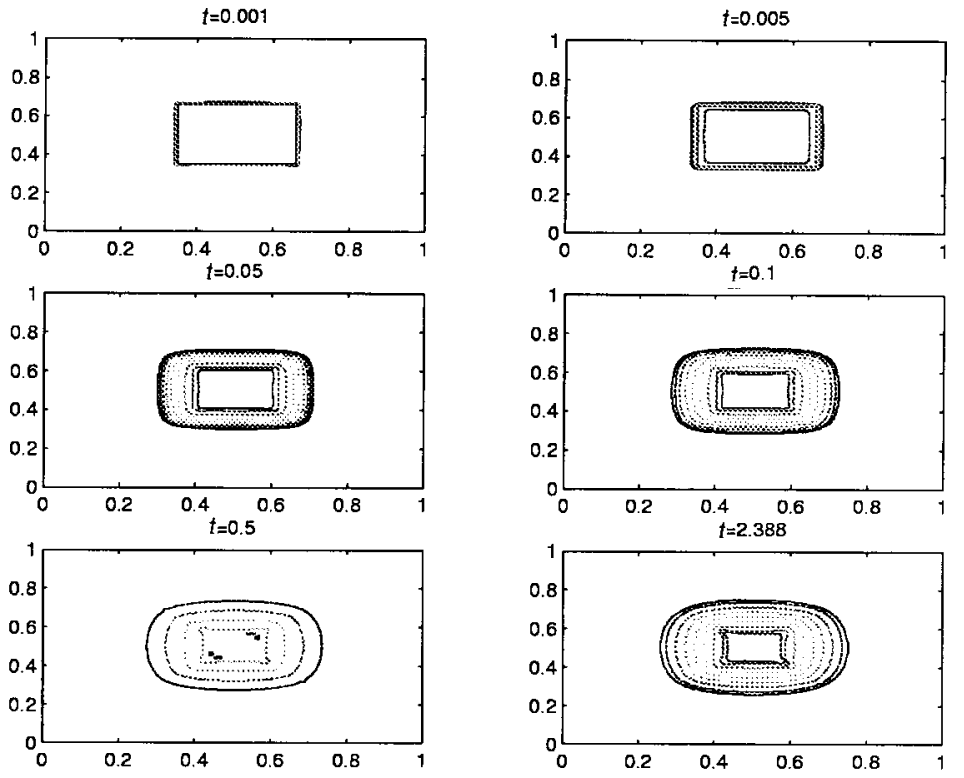

FIG. 10. Evolution of $w$ with pinning, $J_{p}=4, u_{\mathrm{b}}=1 w_{\mathrm{b}}=0$.
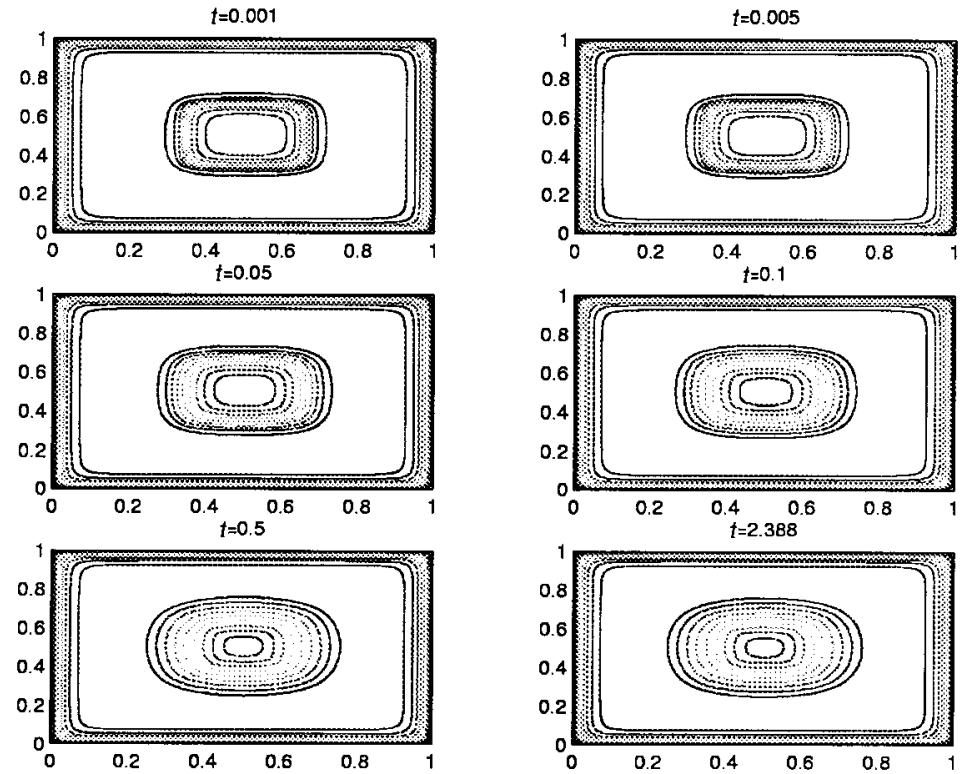

FIG. 11. Evolution of $u$ with pinning, $J_{p}=4, u_{\mathrm{b}}=1 w_{\mathrm{b}}=0$. 

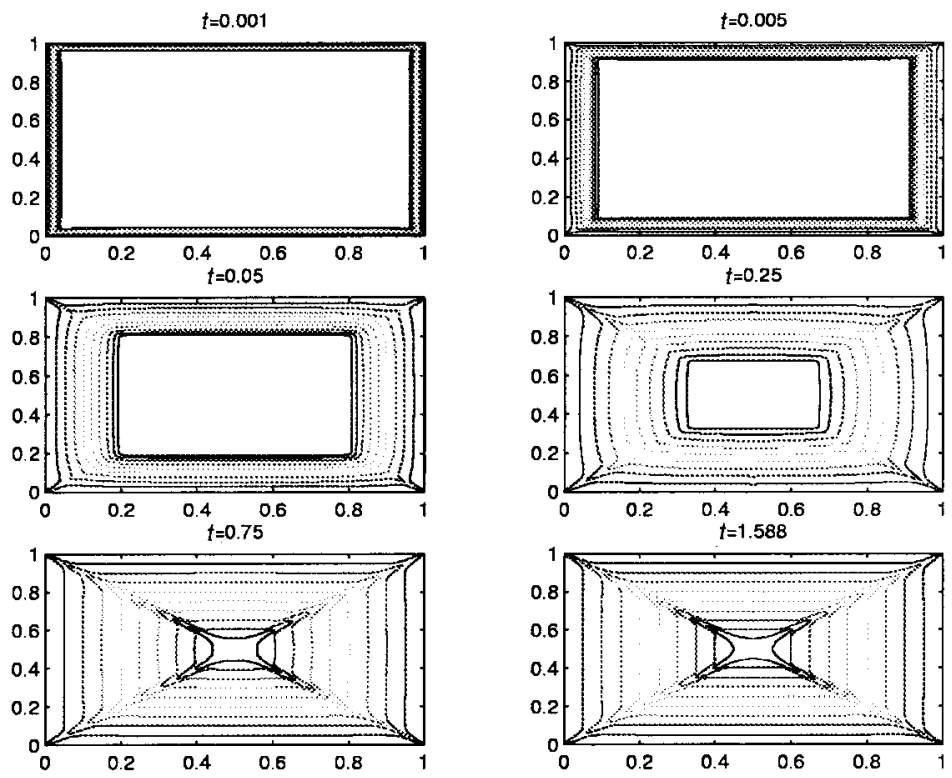

FIG. 12. Evolution of $w$ with nucleation and pinning, $J_{p}=J_{n}=4, u_{\mathrm{b}}=w_{\mathrm{b}}=1$.

$t=0.001$
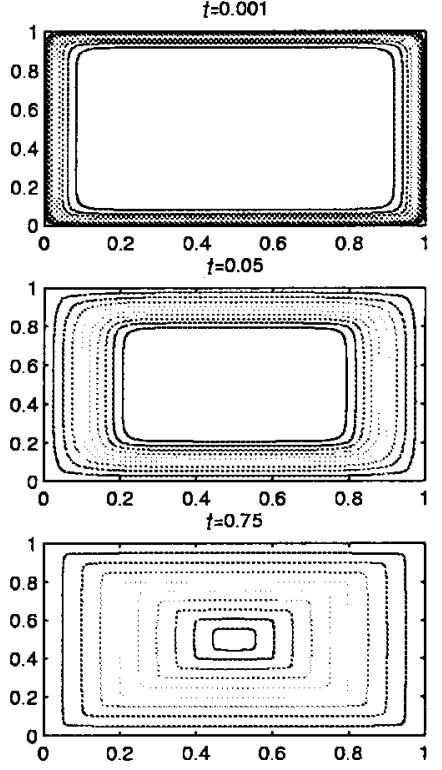

$t=0.005$
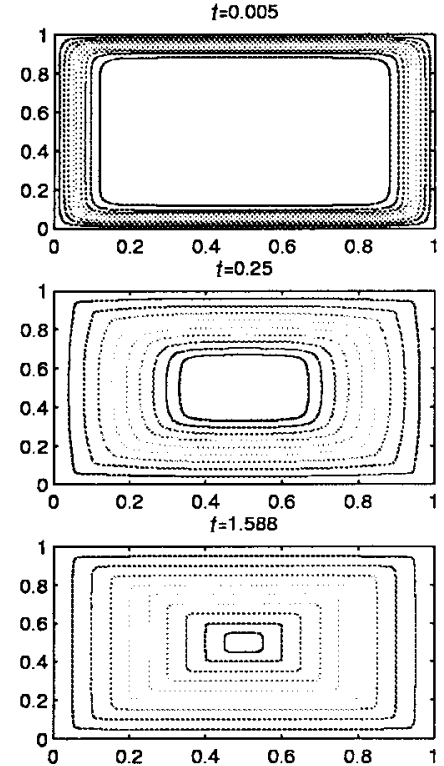

FIG. 13. Evolution of $u$ with nucleation and pinning, $J_{p}=J_{n}=2, u_{\mathrm{b}}=w_{\mathrm{b}}=1$. 

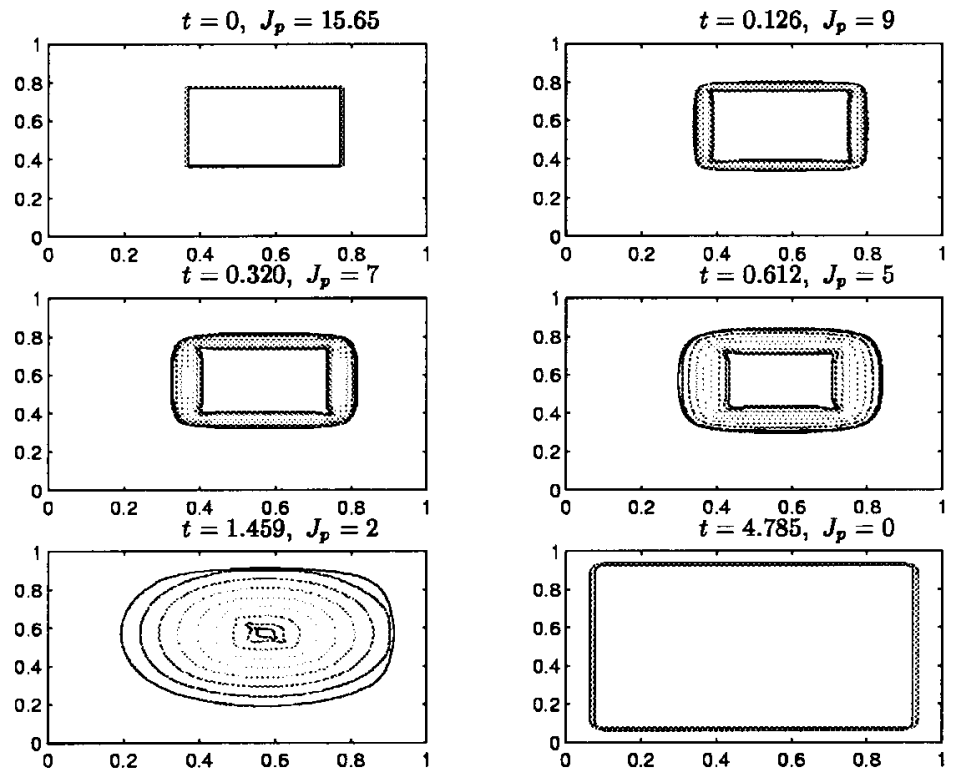

FIG. 14. Steady-state solutions of $w$ with pinning $u_{\mathrm{b}}=1, w_{\mathrm{b}}=0$.
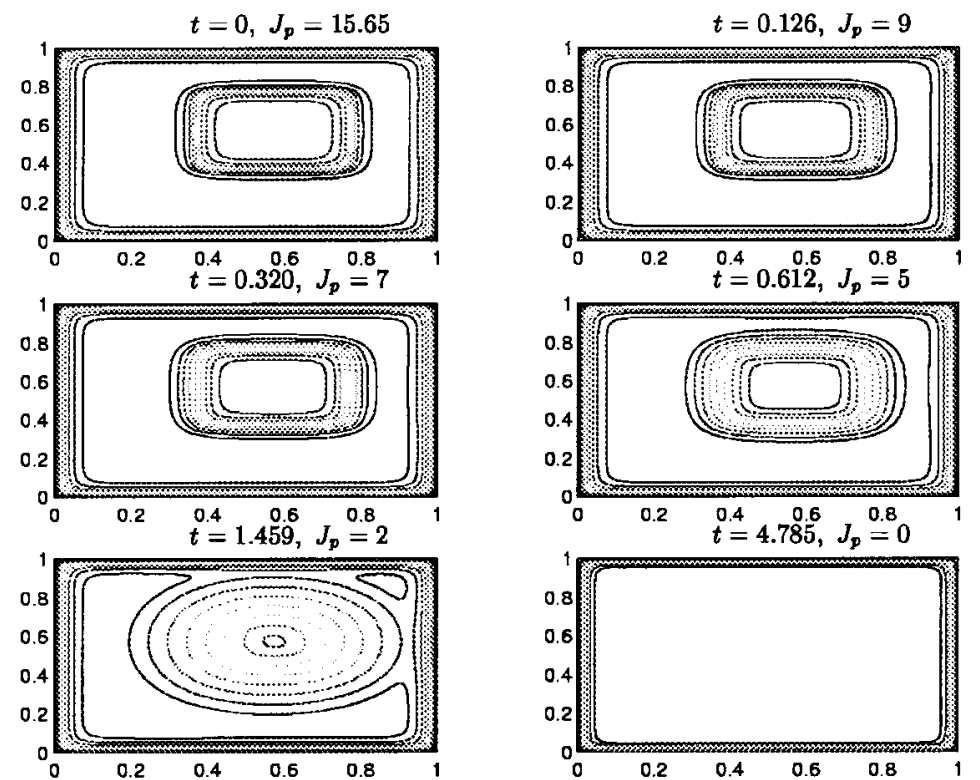

FIG. 15. Steady-state solutions of $u$ with pinning $u_{\mathrm{b}}=1, w_{\mathrm{b}}=0$. 

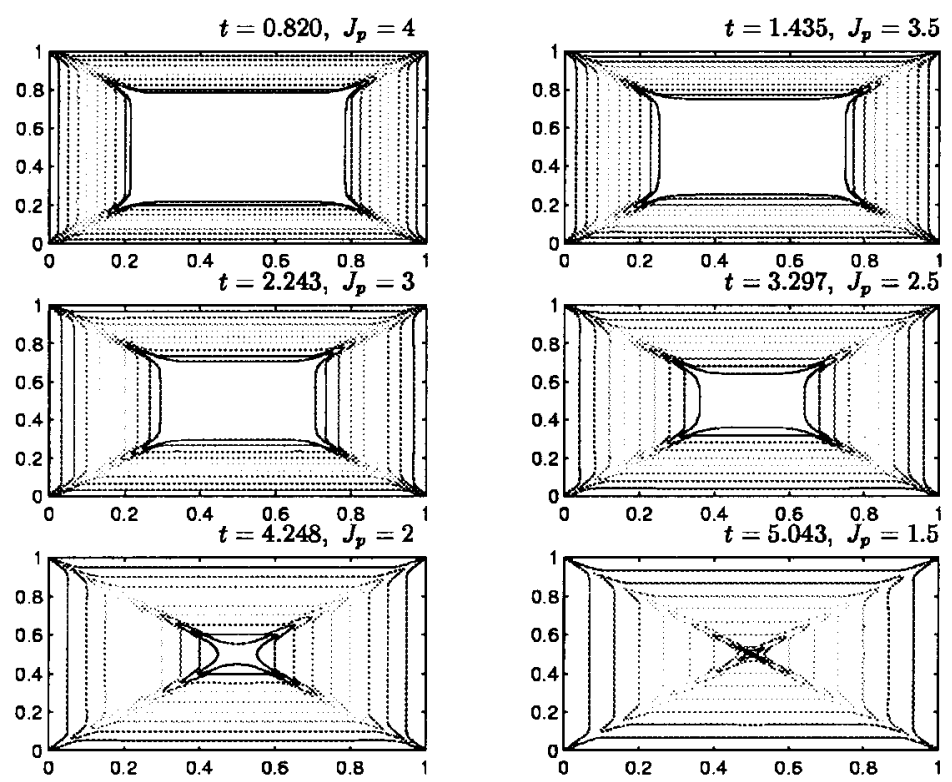

FIG. 16. Steady-state solutions of $w$ with Nucleation and Pinning $u_{\mathrm{b}}=w_{\mathrm{b}}=1$.
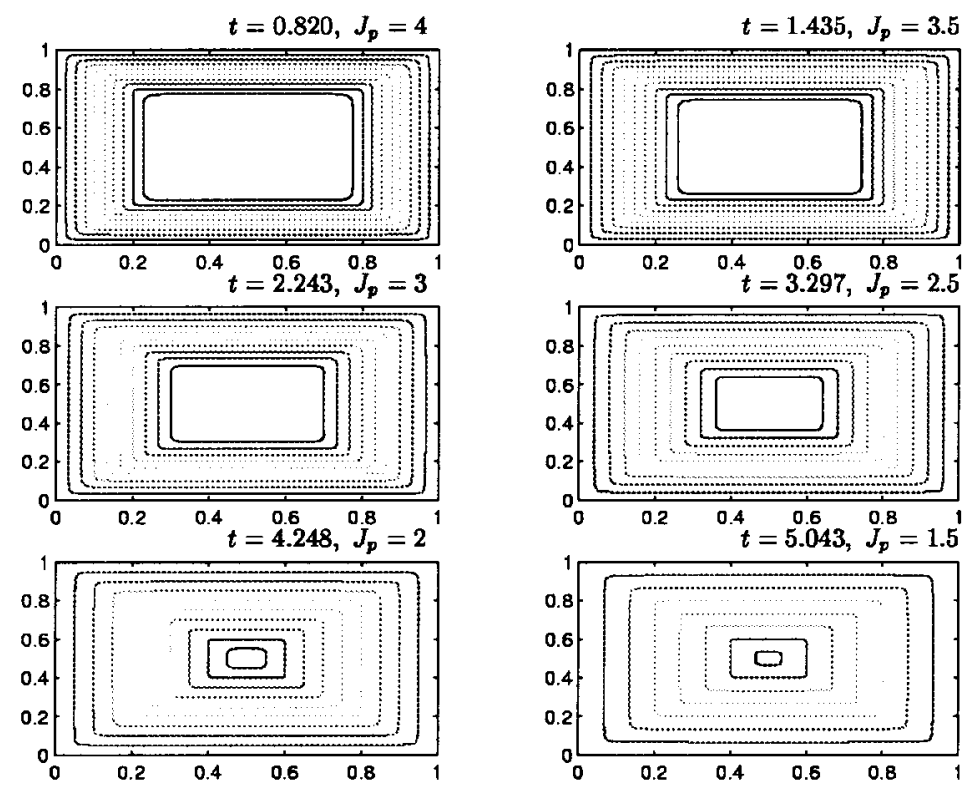

FIG. 17. Steady-state solutions of $u$ with Nucleation and Pinning $u_{\mathrm{b}}=w_{\mathrm{b}}=1$. 
nucleation current $J_{n}$ to be equal to the critical pinning current $J_{p}$. From these figures we see that the vorticity enters the domain at the boundary and then spreads towards the centre of the domain. Furthermore, the smaller the value of $J_{n}=J_{p}$ used, the more vorticity enters the domain and the further to the centre of the domain it spreads. From Figs 12 and 16 we see that the flux lines are constricted along the diagonals of the rectangular cross-section, this effect is due to the finite penetration depth $\lambda$.

In Figs 6 and 7 we see the effects of varying values of $\lambda$. In particular, we note two things, firstly, the absolute value of the gradient of the magnetic field, $\left|u_{x}\right|=J$ increases and secondly, the difference between the vorticity and the magnetic field within the sample decreases. In Fig. 6 we see the effects that occur when the value of $J$ is increased in samples in which no vorticity is nucleated at the boundary, but in which there are pinning sites (i.e. samples in which the nucleation current $J_{n}$ is very large); there become more places in which $J$ exceed the critical pinning current $J_{p}$ and as a result, fewer places where the vorticity is pinned, resulting in the vorticity spreading out more in the domain. Similarly, in Fig. 7 we see the effects that occur when the value of $J$ is increased in samples in which vorticity is nucleated at the boundary and then pinned in the domain; eventually $J$ exceeds the critical nucleation current $J_{n}$ at the boundary and enters the domain, where at certain places it is pinned.

Figures 8 and 9 are presented to enable comparisons between solutions of Bean's model and the mean field model with $\lambda$ small. Comparing the results in Figs 8 and 9 to the critical state Bean's model solutions described for example in [3] we see that solutions of the two models compare favourably. We note that for an increasing applied magnetic field at the boundary, the Bean magnetic field or the vorticity, satisfies

$$
u=\max \left(u_{\mathrm{b}}-J_{p} \operatorname{dist}(x, \partial \Omega), 0\right) .
$$

\section{Conclusion}

In this paper we give a precise formulation of a vortex density model incorporating flux pinning and vortex nucleation at the boundary. We note that the nucleation condition (1.12) was only postulated by Chapman in [4] as a natural choice and was not derived from say the Ginzburg-Landau equations. We show that the problem has a unique solution in one space dimension and we derive a numerical scheme which we show to be convergent. We present some numerical simulations obtained using this scheme with non-zero $\lambda$ and a non-extreme pinning function $f$, whereas numerical studies in the physics literature are related to non-linear diffusion equations (i.e. $\lambda=0$ ) or the classical Bean's model.

This work may be regarded as one of the initial steps in the rigorous mathematical study of vortex density models. Further work in this area could include investigating some of the following;

- the well posedness of the free boundary problem for the motion of a line vortex

- the convergence of the Ginzburg-Landau equations to the aforementioned free boundary line vortex problem

- rigorous mathematical analysis of the well posedness of vortex density models in three dimensions

- convergence of the averaging procedure for the motion of line vortices to vortex density models 
- various asymptotic limits for the vortex density models giving a chain that rigorously links Bean's model to the Ginzburg-Landau equations, for example, in cylindrical geometries with parallel or transverse applied magnetic field.

Finally, a Bean's model suitable for three dimensions might be obtained via a limit of a suitable vortex density model in three dimensions.

\section{REFERENCES}

1. Barrett, J. W. \& Prigozhin, L. Bean's critical-state model as the $p \rightarrow \infty$ limit of an evolutionary $p$-Laplacian equation. J. Nonlinear Anal. (to appear).

2. Bass, F., Shapiro, B. Y., Shapiro, I., \& Shvartser, M. Nonlinear flux diffusion in Type-II superconductors. Physica C 297, (1998) 269-276.

3. Brandt, E. H. The flux-line lattice in superconductors. Rep. Prog. Phys. 58, (1995) 1465-1594.

4. Chapman, S. J. A mean-field model of superconducting vortices in three dimensions. SIAM J. Appl. Math. 55, (1995) 1252-1274.

5. Chapman, S. J. \& Richardson, G. Motion of vortices in Type-II superconductors. SIAM J. Appl. Math. 55, (1995) 1275-1296.

6. Chapman, S. J., Rubinstein, J., \& Schatzman, M. A mean-field model of superconducting vortices. Eur. J. Appl. Math. 7, (1995) 97-111.

7. Ciarlet, P. G. The finite element method for elliptic problems. North-Holland, Amsterdam (1980).

8. Cockburn, B. \& Triandaf, I. Convergence of a finite element method for the drift-diffusion semiconductor device equations: the zero diffusion case. Math. Comput. 59, (1992) 383-401.

9. Elliott, C. M. \& Styles, V. Numerical analysis of a mean field model of superconducting vortices. IMA J. Num. Anal. (to appear).

10. Elliott, C. M., Schätzle, R., \& Stoth, B. E. E. Viscosity solutions of a degenerate parabolicelliptic system arising in the mean-field theory of superconductivity. Arch. Rat. Mech. Anal. 145, (1998) 99-127.

11. Elliott, C. M. \& Styles, V. Flux pinning and boundary nucleation in a mean field model of duperconducting bortices. CMAIA Research Report 99/09, University of Sussex (1999).

12. Gilbarg, D. \& Trudinger, N. S. Elliptic partial differential equations of second order. Springer Verlag, Berlin (1977).

13. Gilchrist, J. Flux diffusion and the porous medium equation. Physica C 191, (1997) 132-142.

14. GodlewSKI, E. \& RAVIART, P. Numerical approximation of hyperbolic systems of conservation laws. Springer, New York (1996).

15. Hale, J. K. Ordinary differential equations. Wiley, New York (1969).

16. Ladyženskaja, O. A., Solonnikov, V. A., \& Ural'Ceva, N. N. Linear and quasi linear equations of parabolic type. American Mathematical Society Providence, Translations of Mathematical Monographs (1968).

17. Markowich, P. A. \& Szmolyan, P. A system of convection-diffusion equations with small diffusion coefficient arising in semiconductor physics. J. Diff. Eqns. 81, (1989) 234-254.

18. Prigozhin, L. On the Bean critical-state model of superconductivity. Eur. J. Appl. Math. 7, (1996) 237-274.

19. Prigozhin, L. The Bean model in superconductivity: variational formulation and numerical solution. $J$. Comput. Phys. 129, (1996) 190-200.

20. SchätZle, R. \& StYles, V. Analysis of a mean field model of superconducting vortices. EJAM 10, (1999) 319-352.

21. VArga, R. S. Matrix iterative analysis. Prentice-Hall, Englewood Cliffs, NJ (1962). 